\title{
Insect gall occurrence in savanna and forest remnant sites of Hidrolândia, GO, Brazil Central
}

\author{
Elienai Cândida e Silva ${ }^{1,3}$; Benedito Baptista dos Santos ${ }^{1,4}$ \& Walter Santos de Araújo $0^{2,5}$ \\ 1 Universidade Federal de Goiás (UFG), Instituto de Ciências Biológicas (ICB), Departamento de Ecologia (DECOL). Goiânia, G0, Brasil. \\ 2 Universidade Estadual de Montes Claros (UNIMONTES), Centro de Ciências Biológicas e da Saúde (CCBS), Departamento de Biologia Geral. \\ Montes Claros, MG, Brasil. \\ 3 E-mail: elienaibio@gmail.com \\ 4 E-mail: benecosantos@yahoo.com.br \\ ${ }^{5}$ ORCID: 0000-0003-0157-6151. E-mail: walterbioaraujo@yahoo.com.br (corresponding author)
}

\begin{abstract}
In this study we perform an inventory of the insect galls in savanna and forest sites of Hidrolândia, Goiás, Brazil. We found 150 insect gall morphotypes, distributed on 39 botanical families and 104 plant species. Among the insect galls, 81 gall morphotypes were recorded in the savanna site and 73 in the forest site. The plant taxa richest in insect galls were the family Fabaceae with 22 gall morphotypes, the genus Bauhinia (Fabaceae) with 15, and the species Siparuna guianensis (Siparunaceae) with seven gall morphotypes. We found gall-inducing insects belonging to orders Diptera, Coleoptera, Lepidoptera and Thysanoptera. The galling insects of family Cecidomyiidae (Diptera) were the most common inducing $48.1 \%$ of the gall morphotypes. This is the first systematic survey of insect galls realized in the city of Hidrolândia, being this the site with the higher insect gall diversity already cataloged to the Central region of Brazil.
\end{abstract}

Key-Words. Cerrado; Fabaceae; Host plants; Insect galls; Plant-insect interaction.

\section{INTRODUCTION}

Galls are abnormal modifications in the vegetal tissues induced mainly by galling insects, and characterized by hypertrophy and hyperplasia of plant cells (Mani, 1964; Shorthouse \& Rohfritsch, 1992). From an evolutionary point of view, insect galls are extended phenotypes of the gall-inducers (Stone \& Schönrogge, 2003), which leads to each species of galling insect induces a specific morphotype of gall on their host plant (Carneiro et al., 2009a). Galls and galling insects are widely distributed around the globe, especially in the Neotropical region (Espírito-Santo \& Fernandes, 2007). In the Neotropics stand out Amazon (Julião et al., 2014), Atlantic Forest (Santos et al., 2014) and Cerrado (Araújo et al., 2014a) as hotspots of insect gall diversity.

The Brazilian Cerrado is composed by a wide variety of vegetation types (Ribeiro \& Walter, 2008), and one of the richest floras of Brazil and in the world (Klink \& Machado, 2005; Mendonça et al., 2008). This great structural and floristic diversity is one of the main explanations for the high insect gall diversity in the Cerrado (Araújo et al., 2014a). In this sense, although the number of insect gall studies in the Cerrado has been growing in the recent years (Araújo et al., 2014a), evidence points that there are still temporal and spatial gaps in the group sampling (e.g., Araújo, 2011; Maia et al., 2014).
To the Central Brazil, insect gall inventories have been published only to the following localities: Caldas Novas (Santos et al., 2012), Goiânia (Santos et al., 2010; Silva et al., 2015), Parque Estadual da Serra dos Pireneus (Araújo et al., 2011), and Parque Nacional das Emas (Araújo et al., 2014b). Given these gaps in knowledge of insect galls in the Central Brazil, in the present study we perform an inventory of the insect galls and their host plants in sites of savanna and forest in $\mathrm{Hi}$ drolândia, Goiás, Central Brazil.

\section{MATERIALS AND METHODS}

The study was realized in the Escola Agrícola (EA) of the Centro de Formação Agroecológico de Hidrolândia (CEFAEH) in the city of Hidrolândia, State of Goiás, Central Brazil $\left(17^{\circ} 00^{\prime} 56^{\prime \prime} S\right.$, $49^{\circ} 12^{\prime} 00^{\prime \prime} \mathrm{W}$; Fig. 1). The climate of region is classified as Aw of Köppen, being humid tropical with wet summer and dry winter. EA-CEFAEH has an area of approximately 40 hectares, most covered by natural vegetation with some level of human disturbance. The area is under Cerrado domain being characterized by several vegetation types, such as grasslands (e.g., Rocky Field), savannas (e.g., Cerrado Sensu Stricto or Neotropical savanna) and forests (e.g., Semidecidual Forest and Gallery Forest) (Ribeiro \& Walter, 2008). 

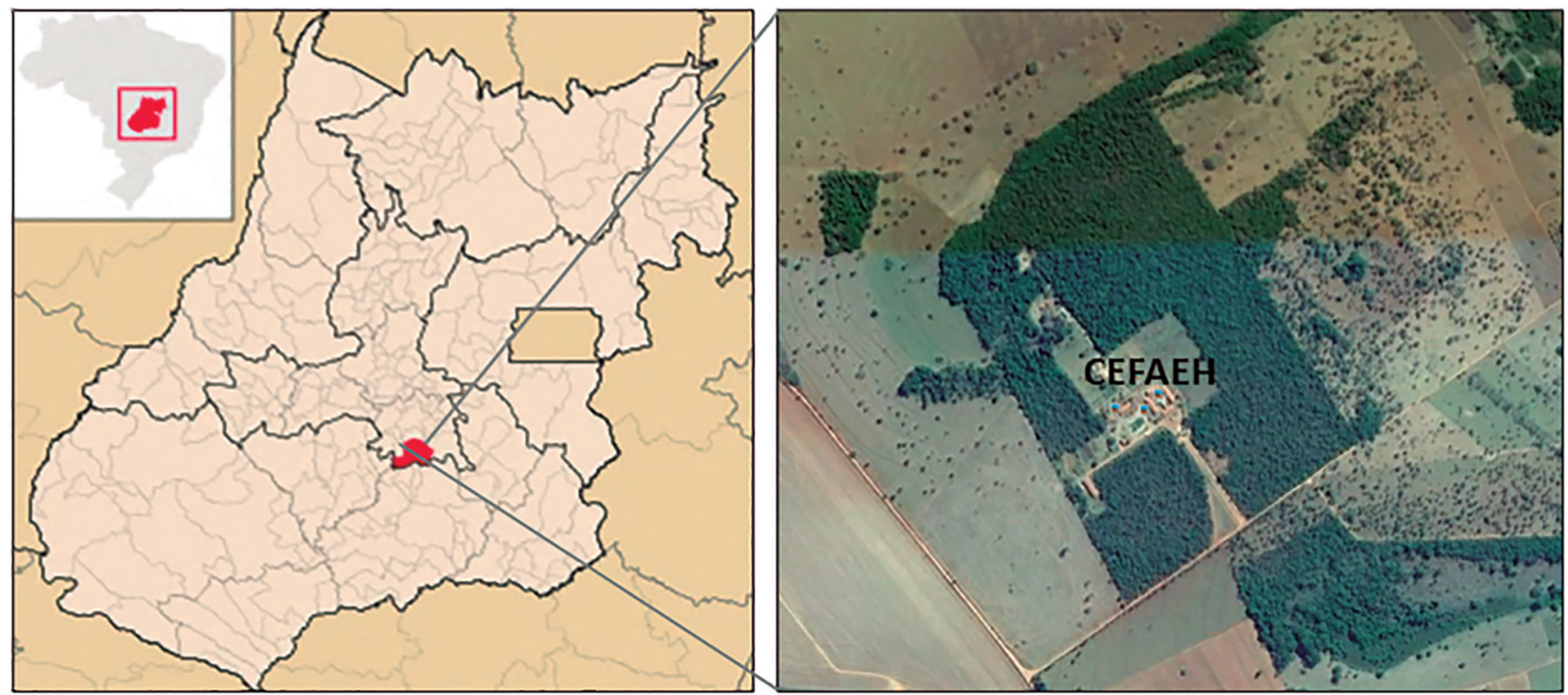

Figure 1. Localization of study area in the CEFAEH, Hidrolândia, Goiás, Central Brazil. Font: Google Earth.

The sampling was done monthly between September 2011 and May 2012 in two vegetation remnants being one of Cerrado Sensu Stricto (hereafter called savanna; $17^{\circ} 01^{\prime} 05^{\prime \prime} S ; 49^{\circ} 11^{\prime} 49^{\prime \prime} W$ ) and other of Semidecidual Forest (hereafter called forest; $17^{\circ} 00^{\prime} 42^{\prime \prime} \mathrm{S} ; 49^{\circ} 12^{\prime} 04^{\prime \prime} \mathrm{W}$ ). We performed the collect of insect galls along two transects (one in each vegetation type) sampled by two hours (Araújo et al., 2011). All host plants had their epigeous parts inspected and all observed insect galls were registered. Samples of each insect galls were photographed, collected and transported individually in labeled plastic bags.

The collected insect galls were taken to the laboratory of Universidade Federal de Goiás (UFG) and packed in plastic container with moistened paper. Insect galls were identified in morphotypes using the host plant species and the gall characteristics (organ of occurrence, form, color, pubescence, and size). In laboratory, the galls were daily observed to verify the emergence of adult insects or dissected to obtainment of immature insects. All obtained insects were fixed in $70 \%$ alcohol and identified using the insect gall literature to Neotropics and Brazil (e.g., Gagné, 1994; Maia \& Fernandes, 2004, Araújo et al., 2011). The identification of the host plant species was made by comparison with the collection of UFG herbarium. We checked plant species nomenclature and synonymy using The Plant List (http://theplantlist.org).

\section{RESULTS AND DISCUSSION}

We found 150 insect gall morphotypes distributed on 39 botanical families and 104 plant species in the EA-CEFAEH (Table 1). Previous studies that inventoried the insect gall diversity in the Cerrado sites ranged from 22 (Urso-Guimarães et al., 2003) to 241 (Carneiro et al., 2009b) gall morphotypes. The insect gall richness recorded in the present study is almost twice of 86.8 morphotypes, which is the average number of gall morphotypes recorded in the different surveys performed in the Brazilian Cerrado (review in Araújo et al., 2014a). For studies in Central region of Brazil, the insect gall richness in the EA-CEFAEH exceeds all previously cataloged sites (Table 2), as for example, the Parque Nacional das Emas that had 97 gall morphotypes recorded (Araújo et al., 2014b). Additionally, the average number of gall morphotypes per host plant species was of 1.44 in the present study, which was very similar to mean of 1.5 listed to Cerrado (Araújo et al., 2014a). These variations in the number of insect gall morphotypes and galls per plant species can be explained by differences in the sampling efforts and number of sampled host plants in the different inventories.

The plant families richest in insect galls were Fabaceae, with 22 gall morphotypes, Malpighiaceae with 13, Sapindaceae with 11, and Erythroxylaceae and Myrtaceae with nine gall morphotypes each (Table 1). Our results are according to previous studies that point Fabaceae as the most important host family of insect galls in the Cerrado (e.g., Maia \& Fernandes, 2004; Santos et al., 2010; Araújo et al., 2011; Santos et al., 2012; Silva et al., 2015). The main explanation for the high insect gall richness hosted by Fabaceae in the Cerrado is its high species number (Southwood, 1960, 1961; Araújo et al., 2014a) of nearly 800 (Mendonça et al., 2008). Malpighiaceae and Myrtaceae also frequently appear in the ranking of insect gall host families most important of Cerrado (Araújo et al., 2014a).

The plant genera Bauhinia (Fabaceae), Erythroxylum (Erythroxylaceae), Myrcia (Myrtaceae), and Siparuna (Siparunaceae) were the most diverse hosts of insect galls with 15, nine, seven, and seven gall morphotypes, respectively (Table 3). The plant species Siparuna guianensis (Siparunaceae), Bauhinia brevipes (Fabaceae), and Erythroxylum sp. (Erythroxylaceae), with seven, six, and five gall morphotypes, respectively, were the most important 
Table 1. Number of host plant species and insect gall morphotypes in the host plant families recorded in EA-CEFAEH, Hidrolândia, Goiás, Brazil.

\begin{tabular}{|c|c|c|c|c|c|}
\hline \multirow{2}{*}{$\begin{array}{l}\text { Host } \\
\text { plant } \\
\text { family }\end{array}$} & \multirow{2}{*}{$\begin{array}{l}\text { Number } \\
\text { of plant } \\
\text { species }\end{array}$} & \multirow{2}{*}{$\begin{array}{l}\text { Number of gall } \\
\text { morphotypes }\end{array}$} & \multicolumn{3}{|c|}{ Gall richness per vegetation types } \\
\hline & & & Savanna & Savanna/Forest & Forest \\
\hline Anacardiaceae & 2 & 2 & 2 & 0 & 0 \\
\hline Annonaceae & 2 & 4 & 2 & 0 & 2 \\
\hline Apocynaceae & 2 & 4 & 2 & 0 & 2 \\
\hline Araliaceae & 1 & 1 & 1 & 0 & 0 \\
\hline Asteraceae & 1 & 2 & 2 & 0 & 0 \\
\hline Bignoniaceae & 1 & 1 & 0 & 0 & 1 \\
\hline Burseraceae & 1 & 2 & 0 & 0 & 2 \\
\hline Caryocaraceae & 1 & 2 & 1 & 1 & 0 \\
\hline Celastraceae & 2 & 2 & 2 & 0 & 0 \\
\hline Combretaceae & 1 & 2 & 2 & 0 & 0 \\
\hline Connaraceae & 3 & 4 & 4 & 0 & 0 \\
\hline Dilleniaceae & 1 & 2 & 2 & 0 & 0 \\
\hline Ebenaceae & 1 & 1 & 1 & 0 & 0 \\
\hline Elaeocarpaceae & 1 & 2 & 0 & 0 & 2 \\
\hline Erythroxylaceae & 3 & 9 & 5 & 0 & 4 \\
\hline Euphorbiaceae & 3 & 4 & 3 & 0 & 1 \\
\hline Fabaceae & 6 & 22 & 17 & 0 & 5 \\
\hline Lamiaceae & 1 & 2 & 2 & 0 & 0 \\
\hline Lauraceae & 2 & 4 & 0 & 0 & 4 \\
\hline Lorantaceae & 1 & 1 & 0 & 0 & 1 \\
\hline Lythraceae & 1 & 1 & 0 & 0 & 1 \\
\hline Malpighiaceae & 6 & 13 & 7 & 0 & 6 \\
\hline Melastomataceae & 3 & 6 & 4 & 0 & 2 \\
\hline Meliaceae & 1 & 3 & 0 & 0 & 3 \\
\hline Myristicaceae & 1 & 1 & 0 & 0 & 1 \\
\hline Myrtaceae & 2 & 9 & 2 & 0 & 7 \\
\hline Nyctaginaceae & 1 & 1 & 1 & 0 & 0 \\
\hline Ochnaceae & 1 & 1 & 1 & 0 & 0 \\
\hline Proteaceae & 1 & 3 & 3 & 0 & 0 \\
\hline Rhamnaceae & 1 & 1 & 0 & 0 & 1 \\
\hline Rubiaceae & 2 & 5 & 0 & 0 & 5 \\
\hline Rutaceae & 1 & 1 & 1 & 0 & 0 \\
\hline Santalaceae & 1 & 1 & 0 & 0 & 1 \\
\hline Sapindaceae & 3 & 11 & 4 & 0 & 7 \\
\hline Siparunaceae & 1 & 7 & 0 & 0 & 7 \\
\hline Smilacaceae & 1 & 3 & 2 & 0 & 1 \\
\hline Styracaceae & 1 & 1 & 0 & 0 & 1 \\
\hline Vitaceae & 3 & 3 & 2 & 0 & 1 \\
\hline Vochysiaceae & 4 & 6 & 3 & 2 & 1 \\
\hline TOTAL & 71 & 150 & 78 & 3 & 69 \\
\hline
\end{tabular}

host plant species. Recent studies have recorded Bauhinia as a very diverse host plant genus of insect galls, which probably is due to genus be very speciose (Costa et al., 2014; Nogueira et al., 2016). The species S. guianensis and B. brevipes also have been previously recorded as important hosts of insect galls (Coelho et al., 2014; Silva et al., 2015). In our study, the most of recorded insect galls occurred on leaves (66.8\%) and stems (24.1\%), which corroborates the pattern of that Neotropical insect galls are more common in leaves and branches (Fernandes et al., 1988; Santos et al., 2010; Araújo et al., 2011). Insect gall morphotypes also vary greatly in form, color and trichome presence or absence (Table 3), being that the most common were globoids (43.3\%), greens (43.3\%) and glabrous (61.1\%). This great variation in the insect gall morphology can be explained by high specificity of gall-inducing insects associated to their host plants (Carneiro et al., 2009a).

In this study we found gall-inducing insects belonging to orders Diptera, Coleoptera, Lepidoptera and Thysanoptera (Table 4). The Figs. 2-8 illustrates the gall morphotypes induced by galling insects. The most common galling taxon was Cecidomyiidae (Diptera), which induced $48.1 \%$ of the insect gall morphotypes. Galling insects of other taxonomic groups were much less frequent (only $7.2 \%$ ), while from $44.7 \%$ of the insect gall morphotypes we did not find taxa of gall-inducers. Our results corroborate previous studies in the Brazilian savannas that point the dominance of Cecidomyiidae (Maia \& Fernandes, 2004; Santos et al., 2010; Araújo et al., 2011), which is considered the main galling insect group of world (Gagné, 2010). The high cecidomyiid diversity in the Cerrado is hypothesized to be due to richest flora and the strongly opportunistic adaptive radiation of group (Araújo et al., 2014a). Besides of gall-inducing insects, we also record several hymenopteran parasitoids from families Braconidae, Encyrtidae, Eulophidae, Eurytomidae, Pteromalidae and Torymidae. Hymenopteran parasitoids are very frequently in Neotropical insect galls (Fernandes \& Santos, 2014) and are the main natural enemies of Cecidomyiidae (Maia \& Azevedo, 2009).

Of the 150 insect gall morphotypes recorded in our study, 81 were recorded in the savanna site and 73 in the forest site. Only the globoid gall morphotype (Gall 18) recorded on Caryocar brasiliense (Caryocaraceae), the conical gall morphotype (Gall 76) of Diplopterys

Table 2. Comparison between the insect gall surveys performed in different areas of Brazil Central.

\begin{tabular}{|c|c|c|c|c|c|c|c|}
\hline \multicolumn{2}{|l|}{ Surveys } & \multicolumn{2}{|r|}{ Sampling } & \multicolumn{3}{|c|}{ Number of recorded taxa } & \multirow[b]{2}{*}{ Reference } \\
\hline Locality & Coordinates & Collect period & Vegetation studied & $\begin{array}{c}\text { Host } \\
\text { plant } \\
\text { families }\end{array}$ & $\begin{array}{c}\text { Host } \\
\text { plant } \\
\text { species }\end{array}$ & $\begin{array}{c}\text { Insect } \\
\text { gall } \\
\text { species }\end{array}$ & \\
\hline EA-CEFAEH & $17^{\circ} 00^{\prime} \mathrm{S}, 49^{\circ} 12^{\prime} \mathrm{W}$ & September 2011 to May 2012 & Semidecidual Forest, Savanna & 39 & 104 & 150 & Present study \\
\hline Campus Samambaia da UFG & $16^{\circ} 36^{\prime} \mathrm{S}, 49^{\circ} 15^{\prime} \mathrm{W}$ & January 2005 to January 2007 & Semidecidual Forest & 12 & 20 & 34 & Santos et al. (2010) \\
\hline Parque Estadual da Serra dos Pireneus & $15^{\circ} 48^{\prime} \mathrm{S}, 48^{\circ} 52^{\prime} \mathrm{W}$ & August 2006 to July 2008 & Gallery and Semidecidual Forest, Savanna & 28 & 51 & 62 & Araújo et al. (2011) \\
\hline Caldas Novas & $17^{\circ} 42^{\prime} \mathrm{S}, 48^{\circ} 38^{\prime} \mathrm{W}$ & November 2008 to August 2009 & Savanna & 21 & 34 & 56 & Santos et al. (2012) \\
\hline Parque Nacional das Emas & $17^{\circ} 49^{\prime} \mathrm{S}, 52^{\circ} 39^{\prime} \mathrm{W}$ & November 2013 & Grassland, Savanna & 24 & 44 & 97 & Araújo et al. (2014b) \\
\hline Mata da Veterinária & $16^{\circ} 36^{\prime} \mathrm{S}, 49^{\circ} 16^{\prime} \mathrm{W}$ & September 2009 to April 2010 & Semidecidual Forest & 20 & 22 & 42 & Silva et al. (2015) \\
\hline
\end{tabular}


Table 3. Host plants, insect gall morphotypes and insect taxa recorded in savanna and forest remnant sites of EA-CEFAEH, Hidrolândia, Goiás, Brazil. Legend: Gall size: $\mathrm{L}=$ Length, $\mathrm{W}=$ Width; Vegetation type: $\mathrm{S}=$ Savanna, $\mathrm{F}=$ Forest.

\begin{tabular}{|c|c|c|c|c|c|c|c|c|c|c|}
\hline \multicolumn{3}{|c|}{ Host plants } & \multicolumn{6}{|c|}{ Insect gall morphotypes } & \multicolumn{2}{|c|}{ Insect taxa } \\
\hline Family & Species & Gall & Organ & Form & Colour & Pubescence & Size $(\mathrm{cm})$ & $\begin{array}{l}\text { Vege- } \\
\text { tation }\end{array}$ & Gall-inducers & $\begin{array}{c}\text { Parasitoids or } \\
\text { inquilines }\end{array}$ \\
\hline Anacardiaceae & Anacardium humile A. St.-Hil. & 1 & Leaf & Conical & Yellow & Glabrous & $\mathrm{L} \pm 0.4 ; \mathrm{W} \pm 0.4$ & $S$ & Cecidomyiidae & \\
\hline Anacardiaceae & Myracrodruon urundeuva M. Allemão & 2 & Leaf & Conical & Brown & Hairy & $\mathrm{L} \pm 0.5 ; \mathrm{W} \pm 0.4$ & $S$ & Cecidomyiidae & \\
\hline Annonaceae & Annonaceae sp. & 3 & Leaf & Discoid & Yellow & Hairy & $L \pm 0.1 ; W \pm 0.1$ & $S$ & Unidentified & \\
\hline Annonaceae & Annonaceae sp. & 4 & Leaf & Discoid & Yellow & Hairy & $\mathrm{L} \pm 0.2 ; \mathrm{W} \pm 0.2$ & $S$ & Unidentified & \\
\hline Annonaceae & Annonaceae sp. & 5 & Stem & Globose & Brown & Glabrous & $L \pm 1.3 ; W \pm 1.3$ & $\mathrm{~F}$ & Unidentified & \\
\hline Annonaceae & Cardiopetalum sp. & 6 & Apical bud & Fusiform & Green & Hairy & $\mathrm{L} \pm 0.3 ; \mathrm{W} \pm 0.2$ & $\mathrm{~F}$ & Unidentified & \\
\hline Apocynaceae & Aspidosperma sp. & 7 & Leaf & Globose & Green & Glabrous & $L \pm 0.3 ; W \pm 0.3$ & $\mathrm{~F}$ & Cecidomyiidae & Pteromalidae \\
\hline Apocynaceae & Aspidosperma sp. & 8 & Leaf & Globose & Green & Glabrous & $\mathrm{L} \pm 0.5 ; \mathrm{W} \pm 0.5$ & $S$ & Cecidomyiidae & \\
\hline Apocynaceae & Aspidosperma sp. & 9 & Stem & Fusiform & Brown & Glabrous & $L \pm 2.0 ; W \pm 0.5$ & $\mathrm{~F}$ & Cecidomyiidae & \\
\hline Apocynaceae & Aspidosperma tomentosum Mart. & 10 & Leaf & Globose & Green & Hairy & $\mathrm{L} \pm 0.3 ; \mathrm{W} \pm 0.3$ & $S$ & Cecidomyiidae & \\
\hline Araliaceae & Schefflera macrocarpa (Cham. \& Schltdl.) Frodin & 11 & Leaf & Globose & Green & Hairy & $\mathrm{L} \pm 0.1 ; \mathrm{W} \pm 0.1$ & $S$ & Cecidomyiidae & \\
\hline Asteraceae & Asteraceae sp. & 12 & Midvein & Globose & White & Hairy & $\mathrm{L} \pm 0.7 ; \mathrm{W} \pm 0.5$ & $S$ & Unidentified & \\
\hline Asteraceae & Asteraceae sp. & 13 & Stem/Petiole & Globose & Green & Glabrous & $L \pm 1.0 ; W \pm 1.0$ & $S$ & Unidentified & \\
\hline Bignoniaceae & Fridericia sp. & 14 & Stem & Fusiform & Brown & Hairy & $\mathrm{L} \pm 1.7 ; \mathrm{W} \pm 0.7$ & $\mathrm{~F}$ & Cecidomyiidae & \\
\hline Burseraceae & Protium sp. & 15 & Leaf & Globose & Yellow & Glabrous & $\mathrm{L} \pm 0.3 ; \mathrm{W} \pm 0.3$ & $\mathrm{~F}$ & Cecidomyiidae & \\
\hline Burseraceae & Protium sp. & 16 & Leaf/Stem/Petiole & Fusiform & Green & Glabrous & $L \pm 0.6 ; W \pm 0.6$ & $\mathrm{~F}$ & Cecidomyiidae & \\
\hline Caryocaraceae & Caryocar brasiliense A. St.-Hil. & 17 & Leaf & Discoid & Yellow & Hairy & $L \pm 0.3 ; W \pm 0.3$ & $S$ & Cecidomyiidae & \\
\hline Caryocaraceae & Caryocar brasiliense A. St.-Hil. & 18 & Leaf & Globose & Yellow & Hairy & $L \pm 0.3 ; W \pm 0.3$ & $S / F$ & Cecidomyiidae & $\begin{array}{l}\text { Eulophidae/ } \\
\text { Eurytomidae/ } \\
\text { Torymidae }\end{array}$ \\
\hline Celastraceae & Celastraceae sp. & 19 & Leaf & Discoid & Green & Glabrous & $L \pm 0.3 ; W \pm 0.3$ & $S$ & Unidentified & \\
\hline Celastraceae & Plenckia populnea Reissek & 20 & Stem & Globose & Brown & Glabrous & $\mathrm{L} \pm 1.5 ; \mathrm{W} \pm 1.5$ & $S$ & Cecidomyiidae & Braconidae \\
\hline Combretaceae & Terminalia argentea Mart. & 21 & Leaf & Discoid & Green & Hairy & $\mathrm{L} \pm 0.3 ; \mathrm{W} \pm 0.3$ & $S$ & Cecidomyiidae & \\
\hline Combretaceae & Terminalia argentea Mart. & 22 & Leaf & Globose & Brown & Hairy & $\mathrm{L} \pm 0.7 ; \mathrm{W} \pm 0.7$ & $S$ & Cecidomyiidae & \\
\hline Connaraceae & Connaraceae sp. & 23 & Inflorescence & Globose & Brown & Glabrous & $\mathrm{L} \pm 0.7 ; \mathrm{W} \pm 0.7$ & $S$ & Cecidomyiidae & Eurytomidae \\
\hline Connaraceae & Connarus sp. & 24 & Leaf & Globose & Brown & Glabrous & $\mathrm{L} \pm 0.5 ; \mathrm{W} \pm 0.5$ & $S$ & Cecidomyiidae & \\
\hline Connaraceae & Connarus sp. & 25 & Stem & Fusiform & Brown & Glabrous & $\mathrm{L} \pm 2.5 ; \mathrm{W} \pm 1.0$ & $S$ & Unidentified & Eulophidae \\
\hline Connaraceae & Connarus suberosus Planch. & 26 & Leaf & Discoid & Green & Glabrous & $\mathrm{L} \pm 0.3 ; \mathrm{W} \pm 0.3$ & $S$ & Cecidomyiidae & \\
\hline Dilleniaceae & Davilla sp. & 27 & Inflorescence & Fusiform & Green & Hairy & $\mathrm{L} \pm 1.0 ; \mathrm{W} \pm 0.5$ & $S$ & Lepidoptera & \\
\hline Dilleniaceae & Davilla sp. & 28 & Leaf & Discoid & Yellow & Glabrous & $\mathrm{L} \pm 0.3 ; \mathrm{W} \pm 0.3$ & $S$ & Cecidomyiidae & \\
\hline Ebenaceae & Diospyros hispida A. DC. & 29 & Apical bud & Globose & Green & Hairy & $\mathrm{L} \pm 3.0 ; \mathrm{W} \pm 3.0$ & $S$ & Lepidoptera & \\
\hline Elaeocarpaceae & Sloanea sp. & 30 & Leaf & Globose & Green & Glabrous & $\mathrm{L} \pm 0.4 ; \mathrm{W} \pm 0.3$ & $\mathrm{~F}$ & Unidentified & \\
\hline Elaeocarpaceae & Sloanea sp. & 31 & Leaf & Globose & Brown & Hairy & $\mathrm{L} \pm 0.4 ; \mathrm{W} \pm 0.4$ & $\mathrm{~F}$ & Unidentified & \\
\hline Erythroxylaceae & Erythroxylum sp. & 32 & Leaf & Amophous & Green & Glabrous & $\mathrm{L} \pm 1.0 ; \mathrm{W} \pm 0.2$ & $S$ & Cecidomyiidae & \\
\hline Erythroxylaceae & Erythroxylum sp. & 33 & Leaf & Discoid & Green & Glabrous & $\mathrm{L} \pm 0.2 ; \mathrm{W} \pm 0.2$ & $\mathrm{~F}$ & Cecidomyiidae & Eulophidae \\
\hline Erythroxylaceae & Erythroxylum sp. & 34 & Leaf & Discoid & Green & Glabrous & $\mathrm{L} \pm 0.4 ; \mathrm{W} \pm 0.4$ & $\mathrm{~F}$ & Cecidomyiidae & \\
\hline Erythroxylaceae & Erythroxylum sp. & 35 & Leaf & Globose & Brown & Hairy & $L \pm 0.6 ; W \pm 0.6$ & $S$ & Cecidomyiidae & Pteromalidae \\
\hline Erythroxylaceae & Erythroxylum sp. & 36 & Leaf & Globose & Brown & Hairy & $\mathrm{L} \pm 0.5 ; \mathrm{W} \pm 0.5$ & $S$ & Cecidomyiidae & \\
\hline Erythroxylaceae & Erythroxylum sp. & 37 & Midvein & Globose & Brown & Hairy & $\mathrm{L} \pm 0.5 ; \mathrm{W} \pm 0.5$ & $\mathrm{~F}$ & Cecidomyiidae & Pteromalidae \\
\hline Erythroxylaceae & Erythroxylum sp. & 38 & Stem & Globose & Brown & Glabrous & $L \pm 0.6 ; W \pm 0.6$ & $\mathrm{~F}$ & Cecidomyiidae & \\
\hline Erythroxylaceae & Erythroxylum suberosum A. St.-Hil. & 39 & Leaf & Globose & Brown & Hairy & $\mathrm{L} \pm 2.0 ; \mathrm{W} \pm 2.0$ & $S$ & $\begin{array}{c}\text { Myrciariamyia } \\
\text { admirabilis Maia \& } \\
\text { Fernandes, } 2007 \\
\text { (Cecidomyiidae) }\end{array}$ & $\begin{array}{l}\text { Encyrtidae/ } \\
\text { Eulophidae/ } \\
\text { Torymidae }\end{array}$ \\
\hline Erythroxylaceae & Erythroxylum tortuosum Mart. & 40 & Leaf & Globose & Green & Glabrous & $\mathrm{L} \pm 0.1 ; \mathrm{W} \pm 0.1$ & $S$ & Cecidomyiidae & \\
\hline Euphorbiaceae & Euphorbiaceae sp. & 41 & Stem/Petiole & Fusiform & Red & Glabrous & $\mathrm{L} \pm 3.0 ; \mathrm{W} \pm 0.5$ & $\mathrm{~F}$ & Unidentified & $\begin{array}{l}\text { Eulophidae/ } \\
\text { Eurytomidae }\end{array}$ \\
\hline Euphorbiaceae & Manihot sp. & 42 & Leaf & Conical & Yellow & Glabrous & $L \pm 1.0 ; W \pm 0.3$ & $S$ & latrophobiasp. & Pteromalidae \\
\hline Euphorbiaceae & Manihot sp. & 43 & Leaf & Conical & Yellow & Glabrous & $\mathrm{L} \pm 0.7 ; \mathrm{W} \pm 0.2$ & $S$ & latrophobiasp. & \\
\hline Euphorbiaceae & Maprounea guianensis Aubl. & 44 & Stem & Fusiform & Red & Glabrous & $L \pm 0.7 ; W \pm 0.5$ & $S$ & Unidentified & \\
\hline Fabaceae & Acosmium dasycarpum (Vogel) Yakovlev & 45 & Leaf & Discoid & Green & Glabrous & $\mathrm{L} \pm 0.5 ; \mathrm{W} \pm 0.5$ & $S$ & Cecidomyiidae & \\
\hline Fabaceae & Bauhinia brevipes Vogel. & 46 & Leaf & Discoid & Yellow & Hairy & $\mathrm{L} \pm 0.1 ; \mathrm{W} \pm 0.1$ & $S$ & Cecidomyiidae & \\
\hline Fabaceae & Bauhinia brevipes Vogel. & 47 & Leaf & Globose & Yellow & Hairy & $\mathrm{L} \pm 0.2 ; \mathrm{W} \pm 0.2$ & $S$ & Cecidomyiidae & \\
\hline Fabaceae & Bauhinia brevipes Vogel. & 48 & Leaf & Globose & Yellow & Hairy & $\mathrm{L} \pm 0.1 ; \mathrm{W} \pm 0.1$ & $S$ & Cecidomyiidae & \\
\hline Fabaceae & Bauhinia brevipes Vogel. & 49 & Leaf & Globose & Brown & Hairy & $\mathrm{L} \pm 0.4 ; \mathrm{W} \pm 0.4$ & $S$ & Cecidomyiidae & \\
\hline Fabaceae & Bauhinia brevipes Vogel. & 50 & Stem & Fusiform & Green & Hairy & $\mathrm{L} \pm 5.5 ; \mathrm{W} \pm 0.6$ & $S$ & Cecidomyiidae & $\begin{array}{c}\text { Eulophidae/ } \\
\text { Braconidae }\end{array}$ \\
\hline
\end{tabular}




\begin{tabular}{|c|c|c|c|c|c|c|c|c|c|c|}
\hline \multicolumn{3}{|c|}{ Host plants } & \multicolumn{6}{|c|}{ Insect gall morphotypes } & \multicolumn{2}{|c|}{ Insect taxa } \\
\hline Family & Species & Gall & Organ & Form & Colour & Pubescence & Size $(\mathrm{cm})$ & $\begin{array}{l}\text { Vege- } \\
\text { tation }\end{array}$ & Gall-inducers & $\begin{array}{l}\text { Parasitoids or } \\
\text { inquilines }\end{array}$ \\
\hline Fabaceae & Bauhinia brevipes Vogel. & 51 & Stem & Fusiform & Brown & Hairy & $\mathrm{L} \pm 1.8 ; \mathrm{W} \pm 0.8$ & $S$ & Cecidomyiidae & \\
\hline Fabaceae & Bauhinia curvula Benth. & 52 & Leaf & Globose & Green & Hairy & $\mathrm{L} \pm 0.8 ; \mathrm{W} \pm 0.4$ & $\mathrm{~s}$ & Cecidomyiidae & \\
\hline Fabaceae & Bauhinia curvula Benth. & 53 & Stem & Fusiform & Brown & Glabrous & $\mathrm{L} \pm 3.0 ; \mathrm{W} \pm 1.0$ & $S$ & Cecidomyiidae & \\
\hline Fabaceae & Bauhinia sp. & 54 & Apical bud & Fusiform & Green & Hairy & $\mathrm{L} \pm 1.0 ; \mathrm{W} \pm 1.3$ & $S$ & Unidentified & \\
\hline Fabaceae & Bauhinia sp. & 55 & Leaf & Fusiform & Green & Hairy & $\mathrm{L} \pm 0.4 ; \mathrm{W} \pm 0.3$ & $S$ & Cecidomyiidae & $\begin{array}{l}\text { Eulophidae/ } \\
\text { Braconidae/ } \\
\text { Pteromalidae }\end{array}$ \\
\hline Fabaceae & Bauhinia sp. & 56 & Leaf & Globose & Green & Hairy & $\mathrm{L} \pm 0.5 ; \mathrm{W} \pm 0.5$ & $S$ & Cecidomyiidae & \\
\hline Fabaceae & Bauhinia sp. & 57 & Leaf & Globose & Yellow & Hairy & $\mathrm{L} \pm 0.2 ; \mathrm{W} \pm 0.2$ & $S$ & Unidentified & \\
\hline Fabaceae & Bauhinia sp. & 58 & Leaf & Globose & Brown & Hairy & $\mathrm{L} \pm 0.2 ; \mathrm{W} \pm 0.2$ & $\mathrm{~F}$ & Unidentified & \\
\hline Fabaceae & Bauhinia sp. & 59 & Stem & Fusiform & Brown & Glabrous & $\mathrm{L} \pm 2.0 ; \mathrm{W} \pm 0.8$ & $\mathrm{~F}$ & Unidentified & Braconidae \\
\hline Fabaceae & Bauhinia sp. & 60 & Stem & Globose & Brown & Hairy & $\mathrm{L} \pm 2.5 ; \mathrm{W} \pm 2.0$ & $\mathrm{~s}$ & Lepidoptera & Torymidae \\
\hline Fabaceae & Fabaceae sp. & 61 & Leaf & Discoid & Red & Glabrous & $\mathrm{L} \pm 0.4 ; \mathrm{W} \pm 0.4$ & $\mathrm{~F}$ & Unidentified & \\
\hline Fabaceae & Fabaceae sp. & 62 & Leaf & Globose & Green & Glabrous & $\mathrm{L} \pm 0.2 ; \mathrm{W} \pm 0.2$ & $\mathrm{~F}$ & Unidentified & \\
\hline Fabaceae & Fabaceae sp. & 63 & Leaf & Globose & Yellow & Glabrous & $\mathrm{L} \pm 0.3 ; \mathrm{W} \pm 0.3$ & $S$ & Unidentified & \\
\hline Fabaceae & Fabaceae sp. & 64 & Leaf & Globose & Green & Hairy & $\mathrm{L} \pm 0.3 ; \mathrm{W} \pm 0.3$ & $S$ & Unidentified & \\
\hline Fabaceae & Fabaceae sp. & 65 & Petiole & Globose & Green & Glabrous & $\mathrm{L} \pm 0.3 ; \mathrm{W} \pm 0.3$ & $\mathrm{~F}$ & Unidentified & \\
\hline Fabaceae & Sclerolobium paniculatum Vogel. & 66 & Leaf & Globose & Green & Hairy & $\mathrm{L} \pm 0.1 ; \mathrm{W} \pm 0.1$ & $\mathrm{~s}$ & Unidentified & \\
\hline Lamiaceae & Lamiaceae sp. & 67 & Midvein & Fusiform & Green & Hairy & $\mathrm{L} \pm 0.4 ; \mathrm{W} \pm 0.2$ & $S$ & Unidentified & \\
\hline Lamiaceae & Lamiaceae sp. & 68 & Stem & Fusiform & Brown & Hairy & $\mathrm{L} \pm 1.5 ; \mathrm{W} \pm 0.5$ & $S$ & Unidentified & \\
\hline Lauraceae & Lauraceae sp. & 69 & Leaf & Globose & Green & Glabrous & $\mathrm{L} \pm 0.5 ; \mathrm{W} \pm 0.5$ & $\mathrm{~F}$ & Unidentified & \\
\hline Lauraceae & Nectandra cuspidata Nees \& Mart. & 70 & Leaf & Discoid & White & Hairy & $\mathrm{L} \pm 0.3 ; \mathrm{W} \pm 0.3$ & $\mathrm{~F}$ & Cecidomyiidae & Eulophidae \\
\hline Lauraceae & Nectandra cuspidata Nees \& Mart. & 71 & Leaf & Discoid & Green & Glabrous & $\mathrm{L} \pm 0.9 ; \mathrm{W} \pm 0.9$ & $\mathrm{~F}$ & Phlaeothripidae & \\
\hline Lauraceae & Nectandra cuspidata Nees \& Mart. & 72 & Leaf & Globose & Brown & Hairy & $\mathrm{L} \pm 0.4 ; \mathrm{W} \pm 0.4$ & $\mathrm{~F}$ & Unidentified & Eulophidae \\
\hline Lorantaceae & Lorantaceae sp. & 73 & Leaf & Discoid & Green & Glabrous & $\mathrm{L} \pm 0.5 ; \mathrm{W} \pm 0.5$ & $\mathrm{~F}$ & Unidentified & \\
\hline Lythraceae & Diplusodon sp. & 74 & Stem/Petiole & Fusiform & White & Glabrous & $\mathrm{L} \pm 1.0 ; \mathrm{W} \pm 0.4$ & $\mathrm{~F}$ & Unidentified & \\
\hline Malpighiaceae & Byrsonima sp. & 75 & Leaf & Conical & Green & Hairy & $\mathrm{L} \pm 0.4 ; \mathrm{W} \pm 0.2$ & $S$ & Cecidomyiidae & \\
\hline Malpighiaceae & $\begin{array}{l}\text { Diplopterys pubipetala (A. Juss.) W.R.Anderson } \\
\& \text { C.Davis }\end{array}$ & 76 & Leaf & Conical & Green & Glabrous & $\mathrm{L} \pm 1.0 ; \mathrm{W} \pm 0.3$ & $S / F$ & Phlaeothripidae & Eulophidae \\
\hline Malpighiaceae & $\begin{array}{l}\text { Diplopterys pubipetala (A. Juss.) W.R.Anderson } \\
\text { \& C.Davis }\end{array}$ & 77 & Leaf & Discoid & Green & Glabrous & $\mathrm{L} \pm 0.5 ; \mathrm{W} \pm 0.5$ & $\mathrm{~F}$ & Unidentified & Eurytomidae \\
\hline Malpighiaceae & $\begin{array}{l}\text { Diplopterys pubipetala (A. Juss.) W.R.Anderson } \\
\& \text { C.Davis }\end{array}$ & 78 & Leaf & Discoid & Green & Glabrous & $\mathrm{L} \pm 0.6 ; \mathrm{W} \pm 0.6$ & $S$ & Unidentified & \\
\hline Malpighiaceae & Heteropterys eglandulosa A. Juss. & 79 & Leaf & Discoid & Yellow & Hairy & $\mathrm{L} \pm 0.3 ; \mathrm{W} \pm 0.3$ & $S$ & Unidentified & \\
\hline Malpighiaceae & Heteropterys sp. & 80 & Leaf & Amophous & Yellow & Glabrous & $\mathrm{L} \pm 1.5 ; \mathrm{W} \pm 0.7$ & $S$ & Unidentified & Eulophidae \\
\hline Malpighiaceae & Malpighiaceae sp. & 81 & Apical bud & Fusiform & Green & Glabrous & $\mathrm{L} \pm 2.5 ; \mathrm{W} \pm 2.0$ & $\mathrm{~F}$ & Unidentified & \\
\hline Malpighiaceae & Malpighiaceae sp. & 82 & Leaf & Discoid & Green & Glabrous & $\mathrm{L} \pm 0.6 ; \mathrm{W} \pm 0.6$ & $\mathrm{~F}$ & Phlaeothripidae & Eulophidae \\
\hline Malpighiaceae & Malpighiaceae sp. & 83 & Stem & Globose & Brown & Glabrous & $\mathrm{L} \pm 2.5 ; \mathrm{W} \pm 2.5$ & $\mathrm{~F}$ & Cecidomyiidae & \\
\hline Malpighiaceae & Malpighiaceae sp. & 84 & Leaf & Globose & Green & Glabrous & $\mathrm{L} \pm 0.5 ; \mathrm{W} \pm 0.5$ & $\mathrm{~F}$ & Unidentified & \\
\hline Malpighiaceae & Malpighiaceae sp. & 85 & Leaf & Globose & Green & Glabrous & $\mathrm{L} \pm 0.5 ; \mathrm{W} \pm 0.4$ & $\mathrm{~F}$ & Unidentified & \\
\hline Malpighiaceae & Malpighiaceae sp. & 86 & Stem & Globose & Brown & Glabrous & $\mathrm{L} \pm 2.0 ; \mathrm{W} \pm 1.5$ & $S$ & Cecidomyiidae & \\
\hline Malpighiaceae & Peixotoasp. & 87 & Leaf & Globose & Brown & Hairy & $\mathrm{L} \pm 0.6 ; \mathrm{W} \pm 0.6$ & $S$ & Cecidomyiidae & Eulophidae \\
\hline Melastomataceae & Melastomataceae sp. & 88 & Leaf/Petiole & Globose & Green & Hairy & $\mathrm{L} \pm 0.5 ; \mathrm{W} \pm 0.5$ & $\mathrm{~F}$ & Unidentified & \\
\hline Melastomataceae & Melastomataceae sp. & 89 & Stem & Fusiform & Brown & Glabrous & $\mathrm{L} \pm 3.5 ; \mathrm{W} \pm 1.3$ & $\mathrm{~s}$ & Unidentified & Torymidae \\
\hline Melastomataceae & Melastomataceae sp. & 90 & Stem & Fusiform & Brown & Glabrous & $\mathrm{L} \pm 5.0 ; \mathrm{W} \pm 2.3$ & $S$ & Unidentified & \\
\hline Melastomataceae & Miconia sp. & 91 & Leaf & Discoid & Green & Hairy & $\mathrm{L} \pm 0.5 ; \mathrm{W} \pm 0.5$ & $\mathrm{~F}$ & Unidentified & \\
\hline Melastomataceae & Miconia sp. & 92 & Leaf & Globose & Brown & Hairy & $\mathrm{L} \pm 0.2 ; \mathrm{W} \pm 0.2$ & $S$ & Lepidoptera & \\
\hline Melastomataceae & Tibouchina sp. & 93 & Stem & Fusiform & Brown & Glabrous & $\mathrm{L} \pm 2.0 ; \mathrm{W} \pm 1.0$ & $S$ & Lepidoptera & \\
\hline Meliaceae & Trichilia sp. & 94 & Leaf & Discoid & Yellow & Glabrous & $\mathrm{L} \pm 0.1 ; \mathrm{W} \pm 0.1$ & $\mathrm{~F}$ & Unidentified & \\
\hline Meliaceae & Trichilia sp. & 95 & Midvein & Fusiform & Yellow & Glabrous & $\mathrm{L} \pm 0.9 ; \mathrm{W} \pm 0.3$ & $\mathrm{~F}$ & Unidentified & \\
\hline Meliaceae & Trichilia sp. & 96 & Stem & Fusiform & Brown & Glabrous & $\mathrm{L} \pm 2.6 ; \mathrm{W} \pm 0.6$ & $\mathrm{~F}$ & Unidentified & Eulophidae \\
\hline Myristicaceae & Myristicaceae sp. & 97 & Leaf & Conical & Brown & Hairy & $\mathrm{L} \pm 0.4 ; \mathrm{W} \pm 0.4$ & $\mathrm{~F}$ & Unidentified & \\
\hline Myrtaceae & Myrcia sp. & 98 & Leaf & Amophous & Yellow & Glabrous & $\mathrm{L} \pm 1.0 ; \mathrm{W} \pm 1.0$ & $\mathrm{~F}$ & Phlaeothripidae & \\
\hline Myrtaceae & Myrcia sp. & 99 & Leaf & Discoid & Green & Glabrous & $\mathrm{L} \pm 0.3 ; \mathrm{W} \pm 0.2$ & $\mathrm{~F}$ & Cecidomyiidae & \\
\hline Myrtaceae & Myrcia sp. & 100 & Leaf & Globose & Green & Hairy & $\mathrm{L} \pm 0.1 ; \mathrm{W} \pm 0.1$ & $\mathrm{~F}$ & Cecidomyiidae & \\
\hline Myrtaceae & Myrcia sp. & 101 & Stem & Fusiform & Brown & Glabrous & $\mathrm{L} \pm 1.0 ; \mathrm{W} \pm 0.5$ & $\mathrm{~F}$ & Unidentified & Eurytomidae \\
\hline Myrtaceae & Myrcia sp. & 102 & Stem & Fusiform & Brown & Glabrous & $\mathrm{L} \pm 2.0 ; \mathrm{W} \pm 1.0$ & $\mathrm{~F}$ & Unidentified & \\
\hline Myrtaceae & Myrcia sp. & 103 & Stem & Fusiform & Brown & Glabrous & $\mathrm{L} \pm 3.5 ; \mathrm{W} \pm 0.7$ & $\mathrm{~F}$ & Unidentified & \\
\hline Myrtaceae & Myrcia sp. & 104 & Stem & Fusiform & Brown & Glabrous & $\mathrm{L} \pm 4.0 ; \mathrm{W} \pm 0.6$ & $\mathrm{~F}$ & Unidentified & \\
\hline Myrtaceae & Myrtaceae sp. & 105 & Leaf bud & Globose & Green & Hairy & $\mathrm{L} \pm 1.3 ; \mathrm{W} \pm 1.3$ & $s$ & Cecidomyiidae & \\
\hline
\end{tabular}




\begin{tabular}{|c|c|c|c|c|c|c|c|c|c|c|}
\hline \multicolumn{3}{|c|}{ Host plants } & \multicolumn{6}{|c|}{ Insect gall morphotypes } & \multicolumn{2}{|c|}{ Insect taxa } \\
\hline Family & Species & Gall & Organ & Form & Colour & Pubescence & Size $(\mathrm{cm})$ & $\begin{array}{l}\text { Vege- } \\
\text { tation }\end{array}$ & Gall-inducers & $\begin{array}{c}\text { Parasitoids or } \\
\text { inquilines }\end{array}$ \\
\hline Myrtaceae & Myrtaceae sp. & 106 & Stem & Globose & Brown & Glabrous & $\mathrm{L} \pm 0.5 ; \mathrm{W} \pm 0.5$ & $S$ & Unidentified & \\
\hline Nyctaginaceae & Guapira sp. & 107 & Leaf & Discoid & Red & Glabrous & $\mathrm{L} \pm 0.5 ; \mathrm{W} \pm 0.5$ & $S$ & Cecidomyiidae & Eulophidae \\
\hline 0chnaceae & Ouratea hexasperma (A. St.-Hil.) Baill. & 108 & Leaf & Discoid & Red & Glabrous & $\mathrm{L} \pm 0.3 ; \mathrm{W} \pm 0.3$ & $S$ & Cecidomyiidae & \\
\hline Proteaceae & Roupala montana Aubl. & 109 & Leaf & Conical & Green & Hairy & $\mathrm{L} \pm 0.7 ; \mathrm{W} \pm 0.3$ & $S$ & Cecidomyiidae & Eulophidae \\
\hline Proteaceae & Roupala Montana Aubl. & 110 & Leaf & Discoid & Green & Hairy & $\mathrm{L} \pm 0.4 ; \mathrm{W} \pm 0.4$ & $S$ & Cecidomyiidae & \\
\hline Proteaceae & Roupala montana Aubl. & 111 & Stem & Globose & Brown & Glabrous & $\mathrm{L} \pm 1.3 ; \mathrm{W} \pm 1.3$ & $S$ & Lepidoptera & \\
\hline Rhamnaceae & Rhamnidium sp. & 112 & Lateral bud & Globose & Brown & Glabrous & $\mathrm{L} \pm 1.0 ; \mathrm{W} \pm 0.7$ & $\mathrm{~F}$ & Unidentified & \\
\hline Rubiaceae & Landia sp. & 113 & Leaf & Fusiform & Green & Glabrous & $\mathrm{L} \pm 0.8 ; \mathrm{W} \pm 0.3$ & $\mathrm{~F}$ & Unidentified & \\
\hline Rubiaceae & Rubiaceae sp. & 114 & Leaf & Fusiform & Yellow & Hairy & $\mathrm{L} \pm 0.2 ; \mathrm{W} \pm 0.1$ & $\mathrm{~F}$ & Unidentified & \\
\hline Rubiaceae & Rubiaceae sp. & 115 & Leaf & Globose & Green & Glabrous & $\mathrm{L} \pm 0.1 ; \mathrm{W} \pm 0.1$ & $\mathrm{~F}$ & Unidentified & \\
\hline Rubiaceae & Rubiaceae sp. & 116 & Leaf & Globose & Yellow & Glabrous & $\mathrm{L} \pm 0.3 ; \mathrm{W} \pm 0.3$ & $\mathrm{~F}$ & Unidentified & \\
\hline Rubiaceae & Rubiaceae sp. & 117 & Stem & Fusiform & Brown & Glabrous & $\mathrm{L} \pm 1.2 ; \mathrm{W} \pm 0.6$ & $\mathrm{~F}$ & Unidentified & \\
\hline Rutaceae & Zanthoxylum sp. & 118 & Leaf & Discoid & Green & Hairy & $L \pm 0.1 ; W \pm 0.1$ & $S$ & Unidentified & \\
\hline Santalaceae & Phoradendron sp. & 119 & Leaf & Discoid & Green & Glabrous & $\mathrm{L} \pm 0.2 ; \mathrm{W} \pm 0.2$ & $\mathrm{~F}$ & Unidentified & \\
\hline Sapindaceae & Paullinia sp. & 120 & Axilary bud & Globose & Green & Hairy & $\mathrm{L} \pm 0.7 ; \mathrm{W} \pm 0.7$ & $\mathrm{~F}$ & Unidentified & Eulophidae \\
\hline Sapindaceae & Paullinia sp. & 121 & Stem & Conical & Red & Hairy & $\mathrm{L} \pm 0.3 ; \mathrm{W} \pm 0.2$ & $\mathrm{~F}$ & Unidentified & \\
\hline Sapindaceae & Sapindaceae sp. & 122 & Leaf & Discoid & Yellow & Glabrous & $L \pm 0.3 ; W \pm 0.3$ & $\mathrm{~F}$ & Unidentified & \\
\hline Sapindaceae & Sapindaceae sp. & 123 & Leaf & Globose & Green & Glabrous & $\mathrm{L} \pm 0.3 ; \mathrm{W} \pm 0.3$ & $S$ & Unidentified & \\
\hline Sapindaceae & Sapindaceae sp. & 124 & Leaf & Globose & Green & Glabrous & $\mathrm{L} \pm 0.2 ; \mathrm{W} \pm 0.2$ & $S$ & Unidentified & \\
\hline Sapindaceae & Sapindaceae sp. & 125 & Stem & Conical & Green & Glabrous & $\mathrm{L} \pm 1.3 ; \mathrm{W} \pm 0.5$ & $\mathrm{~F}$ & Unidentified & Eulophidae \\
\hline Sapindaceae & Serjania sp. & 126 & Leaf & Discoid & Green & Glabrous & $\mathrm{L} \pm 0.8 ; \mathrm{W} \pm 0.2$ & $\mathrm{~F}$ & Cecidomyiidae & \\
\hline Sapindaceae & Serjania sp. & 127 & Leaf & Discoid & Green & Glabrous & $\mathrm{L} \pm 0.5 ; \mathrm{W} \pm 0.5$ & $S$ & Cecidomyiidae & \\
\hline Sapindaceae & Serjania sp. & 128 & Leaf & Discoid & Yellow & Glabrous & $L \pm 0.5 ; W \pm 0.5$ & $S$ & Cecidomyiidae & \\
\hline Sapindaceae & Serjania sp. & 129 & Stem & Fusiform & Brown & Hairy & $\mathrm{L} \pm 2.5 ; \mathrm{W} \pm 1.5$ & $\mathrm{~F}$ & Cecidomyiidae & Eurytomidae \\
\hline Sapindaceae & Serjania sp. & 130 & Tendril & Fusiform & Brown & Glabrous & $\mathrm{L} \pm 2.0 ; \mathrm{W} \pm 1.7$ & $\mathrm{~F}$ & Cecidomyiidae & \\
\hline Siparunaceae & Siparuna guianensis Aubl. & 131 & Leaf & Discoid & Green & Glabrous & $L \pm 0.5 ; W \pm 0.5$ & $\mathrm{~F}$ & Cecidomyiidae & \\
\hline Siparunaceae & Siparuna guianensis Aubl. & 132 & Leaf & Fusiform & Green & Glabrous & $\mathrm{L} \pm 0.6 ; \mathrm{W} \pm 0.4$ & $\mathrm{~F}$ & Cecidomyiidae & \\
\hline Siparunaceae & Siparuna guianensis Aubl. & 133 & Midvein & Fusiform & Green & Glabrous & $\mathrm{L} \pm 0.5 ; \mathrm{W} \pm 0.3$ & $\mathrm{~F}$ & Cecidomyiidae & \\
\hline Siparunaceae & Siparuna guianensis Aubl. & 134 & Petiole & Globose & Brown & Glabrous & $\mathrm{L} \pm 0.4 ; \mathrm{W} \pm 0.4$ & $\mathrm{~F}$ & Cecidomyiidae & \\
\hline Siparunaceae & Siparuna guianensis Aubl. & 135 & Stem & Fusiform & Green & Glabrous & $\mathrm{L} \pm 1.0 ; \mathrm{W} \pm 0.7$ & $\mathrm{~F}$ & Cecidomyiidae & \\
\hline Siparunaceae & Siparuna guianensis Aubl. & 136 & Stem & Globose & Brown & Glabrous & $\mathrm{L} \pm 0.4 ; \mathrm{W} \pm 0.4$ & $\mathrm{~F}$ & Cecidomyiidae & Eurytomidae \\
\hline Siparunaceae & Siparuna guianensis Aubl. & 137 & Stem/Petiole & Fusiform & Green & Glabrous & $\mathrm{L} \pm 2.0 ; \mathrm{W} \pm 0.6$ & $\mathrm{~F}$ & Cecidomyiidae & \\
\hline Smilacaceae & Smilax sp. & 138 & Leaf & Globose & Yellow & Glabrous & $\mathrm{L} \pm 0.2 ; \mathrm{W} \pm 0.2$ & $S$ & Unidentified & $\begin{array}{l}\text { Eulophidae/ } \\
\text { Torymidae }\end{array}$ \\
\hline Smilacaceae & Smilax sp. & 139 & Leaf & Globose & Green & Glabrous & $\mathrm{L} \pm 0.6 ; \mathrm{W} \pm 0.6$ & $S$ & Unidentified & \\
\hline Smilacaceae & Smilax sp. & 140 & Leaf/Stem & Fusiform & White & Glabrous & $\mathrm{L} \pm 2.5 ; \mathrm{W} \pm 2.0$ & $\mathrm{~F}$ & Unidentified & \\
\hline Styracaceae & Styrax pohlii A. DC. & 141 & Leaf & Discoid & Yellow & Glabrous & $L \pm 0.1 ; W \pm 0.1$ & $\mathrm{~F}$ & Cecidomyiidae & \\
\hline Vitaceae & Cissus erosa Rich. & 142 & Stem & Globose & Brown & Glabrous & $\mathrm{L} \pm 4.0 ; \mathrm{W} \pm 3.3$ & $S$ & Curculionidae & \\
\hline Vitaceae & Cissus sp. & 143 & Leaf & Globose & Green & Glabrous & $\mathrm{L} \pm 0.4 ; \mathrm{W} \pm 0.4$ & $\mathrm{~F}$ & Unidentified & \\
\hline Vitaceae & Vitaceae sp. & 144 & Stem/Petiole & Globose & Green & Hairy & $\mathrm{L} \pm 0.4 ; \mathrm{W} \pm 0.4$ & $S$ & Unidentified & \\
\hline Vochysiaceae & Qualea dichotoma (Mart.) Warm. ex Wille & 145 & Leaf & Globose & Green & Hairy & $\mathrm{L} \pm 0.2 ; \mathrm{W} \pm 0.2$ & $\mathrm{~F}$ & Cecidomyiidae & \\
\hline Vochysiaceae & Qualea grandiflora Mart. & 146 & Leaf & Discoid & Yellow & Glabrous & $\mathrm{L} \pm 0.3 ; \mathrm{W} \pm 0.3$ & $S$ & Cecidomyiidae & \\
\hline Vochysiaceae & Qualea multiflora Mart. & 147 & Leaf & Globose & Green & Hairy & $\mathrm{L} \pm 0.4 ; \mathrm{W} \pm 0.4$ & $\mathrm{~F} / \mathrm{S}$ & Cecidomyiidae & Eulophidae \\
\hline Vochysiaceae & Qualea multiflora Mart. & 148 & Leaf & Globose & Yellow & Hairy & $\mathrm{L} \pm 0.6 ; \mathrm{W} \pm 0.6$ & $F / S$ & Cecidomyiidae & $\begin{array}{l}\text { Braconidae/ } \\
\text { Eulophidae }\end{array}$ \\
\hline Vochysiaceae & Qualea parviflora Mart. & 149 & Leaf & Discoid & Green & Glabrous & $\mathrm{L} \pm 0.2 ; \mathrm{W} \pm 0.2$ & $S$ & Cecidomyiidae & \\
\hline Vochysiaceae & Qualea parviflora Mart. & 150 & Leaf & Globose & Yellow & Glabrous & $L \pm 0.4 ; W \pm 0.4$ & $S$ & Cecidomyiidae & Encyrtidae \\
\hline
\end{tabular}

Table 4. Number and percentage of insect gall morphotypes in the different taxa of galling insects recorded in EA-CEFAEH, Hidrolândia, Goiás, Brazil.

\begin{tabular}{lcc}
\hline \multirow{2}{*}{ Galling insect taxa } & \multicolumn{2}{c}{ Insect gall morphotypes } \\
\cline { 2 - 3 } & $\mathbf{N}$ & $\%$ \\
\hline Cecidomyiidae (Diptera) & 72 & 48.1 \\
Lepidoptera & 6 & 4.0 \\
Thysanoptera (Phlaeothripidae) & 4 & 2.6 \\
Coleoptera & 1 & 0.6 \\
Unidentified & 67 & 44.7 \\
\hline TOTAL & $\mathbf{1 5 0}$ & $\mathbf{1 0 0}$ \\
\hline
\end{tabular}

pubipetala (Malpighiaceae) and the two gall morphotypes (Galls 147 and 148) recorded on Qualea multiflora (Vochysiaceae) were common between savanna and forest sites. Comparisons between different vegetation types in the Cerrado have pointed higher insect gall richness in the sclerophyllous habitats, with savannas often have greater species richness than the forests (Gonçalves-Alvim \& Fernandes, 2001; Araújo et al., 2011; Araújo et al., 2014a). Our results corroborate the pattern of higher frequency of insect galls in xeric habitats than 

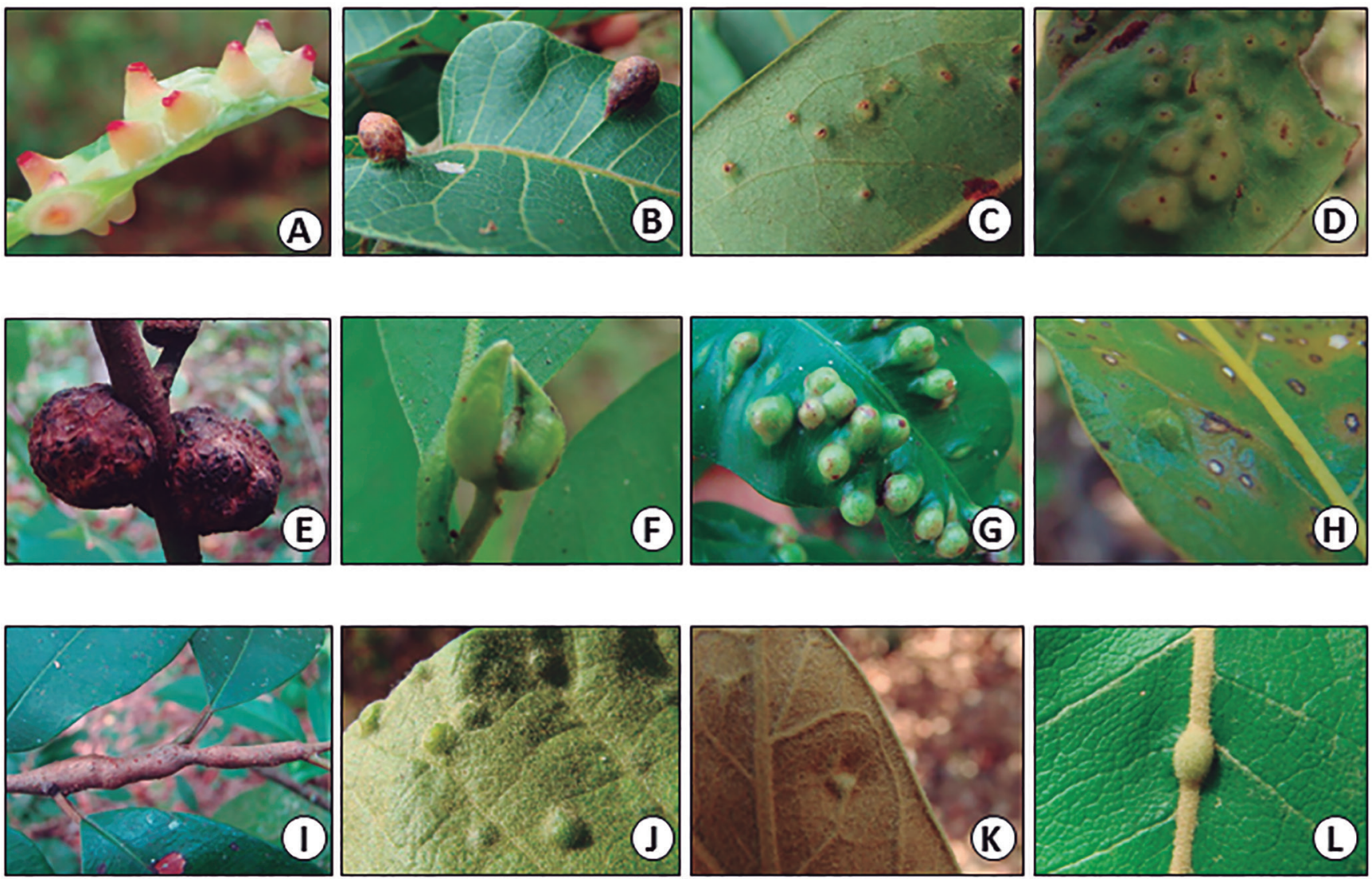

(L)
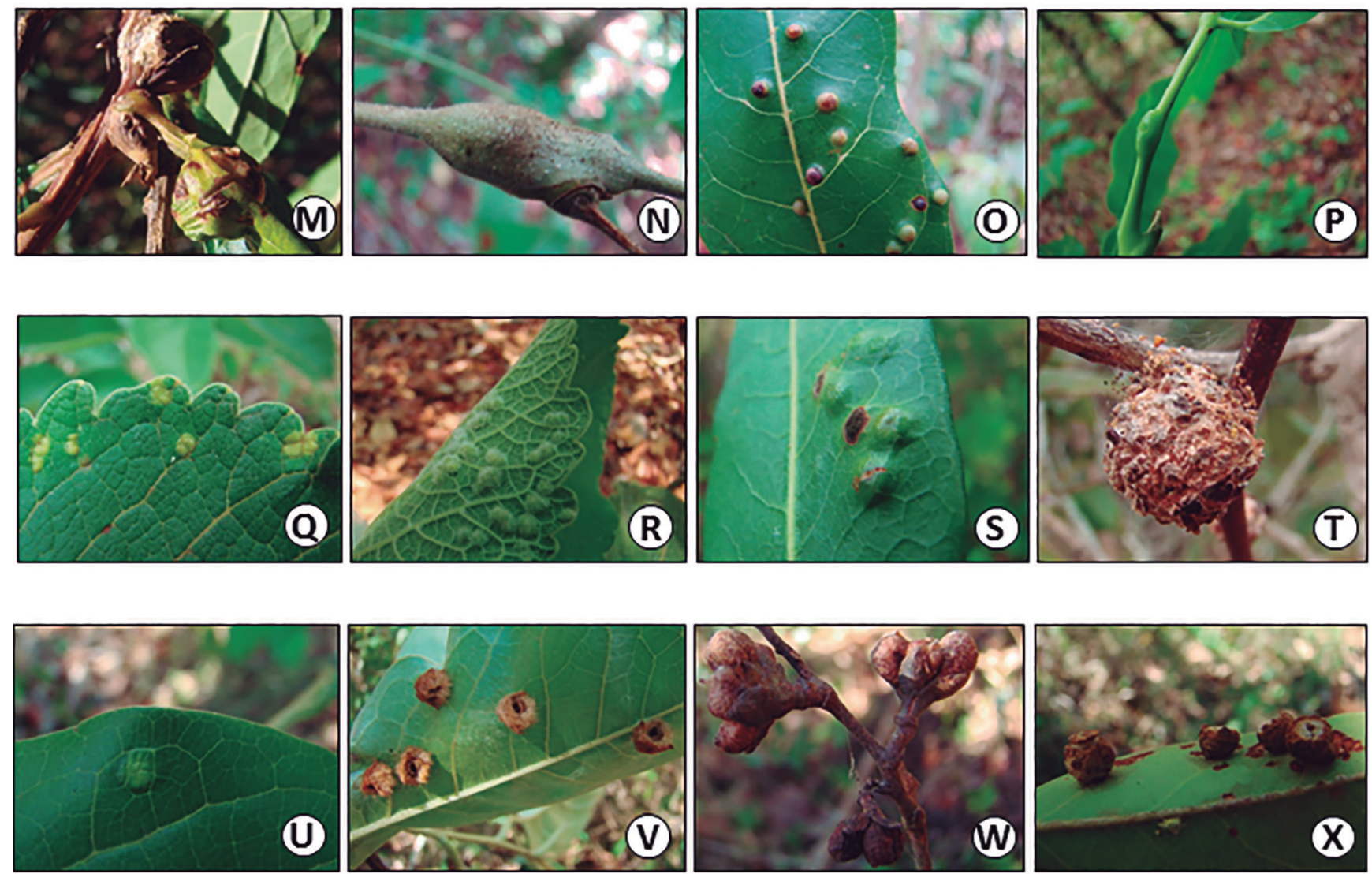

Figure 2. Insect gall morphotypes recorded in EA-CEFAEH, Hidrolândia, Goiás, Brazil. (A) Anacardium humile (Gall 1), (B) Myracrodruon urundeuva (Gall 2), (C) Annonaceae sp. (Gall 3), (D) Annonaceae sp. (Gall 4), (E) Annonaceae sp. (Gall 5), (F) Cardiopetalum sp. (Gall 6), (G) Aspidosperma sp. (Gall 7), (H) Aspidosperma sp. (Gall 8), (I) Aspidosperma sp. (Gall 9), (J) Aspidosperma tomentosum (Gall 10), (K) Schefflera macrocarpa (Gall 11), (L) Asteraceae sp. (Gall 12), (M) Asteraceae sp. (Gall 13), (N) Fridericia sp. (Gall 14), (0) Protium sp. (Gall 15), (P) Protium sp. (Gall 16), (Q) Caryocar brasiliense (Gall 17), (R) C. brasiliense (Gall 18), (S) Celastraceae sp. (Gall 19), (T) Plenckia populnea (Gall 20), (U) Terminalia argentea Mart. (Gall 21), (V) T. argentea (Gall 22), (W) Connaraceae sp. (Gall 23), (X) Connarus sp. (Gall 24). 

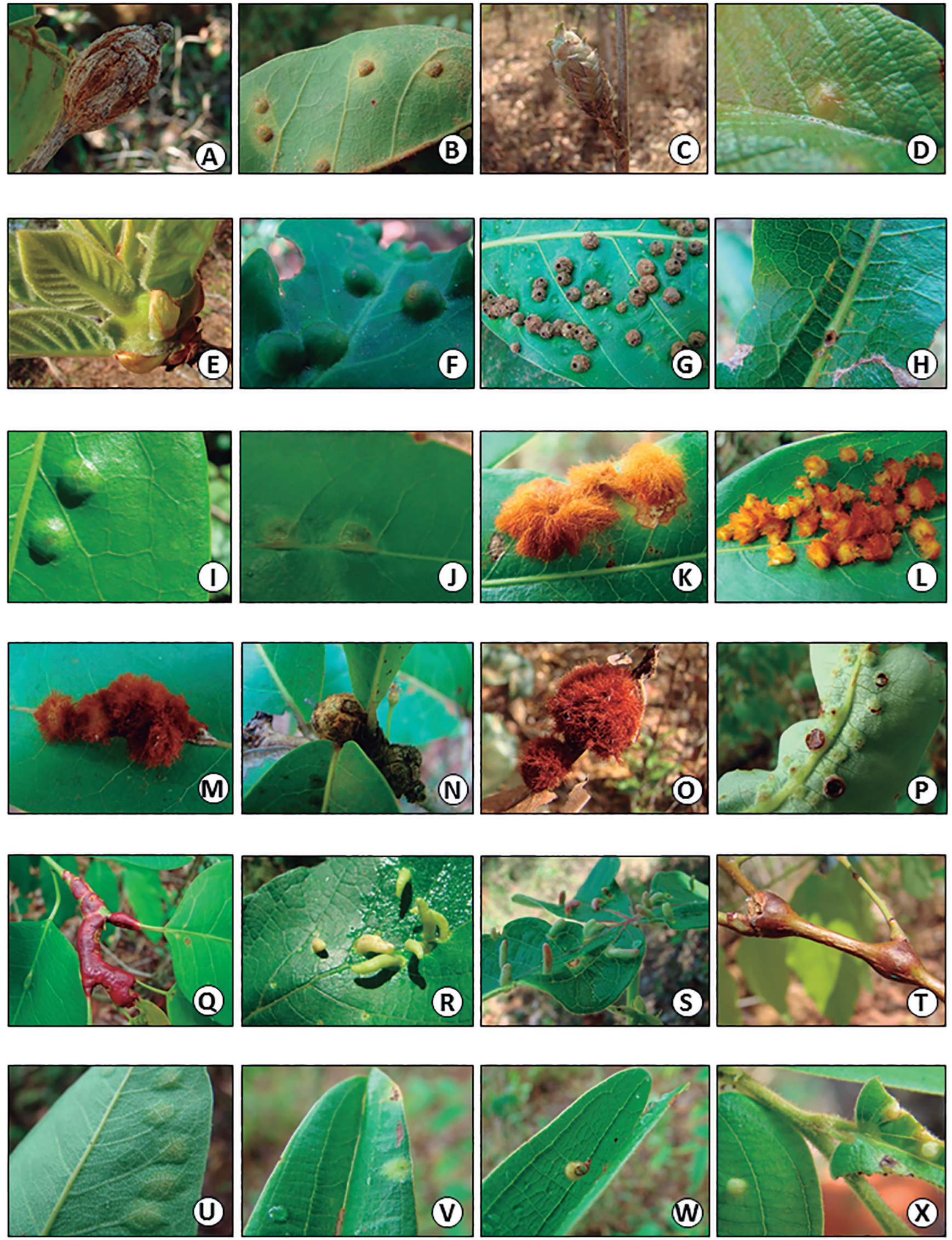

Figure 3. Insect gall morphotypes recorded in EA-CEFAEH, Hidrolândia, Goiás, Brazil. (A) Connarus sp. (Gall 25), (B) Connarus suberosus (Gall 26), (C) Davilla sp. (Gall 27), (D) Davilla sp. (Gall 28), (E) Diospyros hispida (Gall 29), (F) Sloanea sp. (Gall 30), (G) Sloanea sp. (Gall 31), (H) Erythroxylum sp. (Gall 32), (I) Erythroxylum sp. (Gall 33), (J) Erythroxylum sp. (Gall 34), (K) Erythroxylum sp. (Gall 35), (L) Erythroxylum sp. (Gall 36), (M) Erythroxylum sp. (Gall 37), (N) Erythroxylum sp. (Gall 38), (0) Erythroxylum suberosum (Gall 39), (P) Erythroxylum tortuosum (Gall 40), (Q) Euphorbiaceae sp. (Gall 41), (R) Manihot sp. (Gall 42), (S) Manihot sp. (Gall 43), (T) Maprounea guianensis (Gall 44), (U) Acosmium dasycarpum (Gall 45), (V) Bauhinia brevipes (Gall 46), (W) B. brevipes (Gall 47), (X) B. brevipes (Gall 48). 

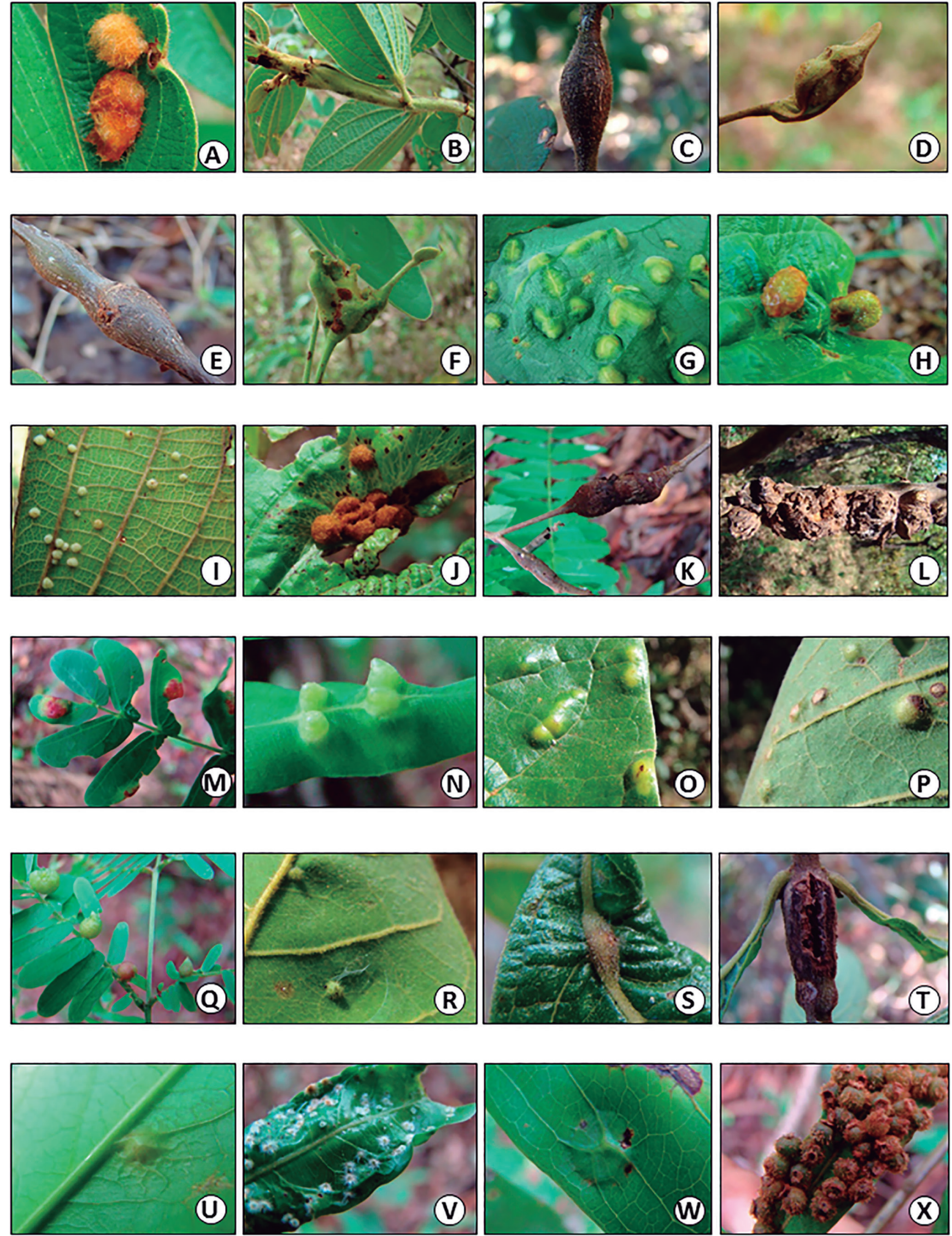

Figure 4. Insect gall morphotypes recorded in EA-CEFAEH, Hidrolândia, Goiás, Brazil. (A) B. brevipes (Gall 49), (B) B. brevipes (Gall 50), (C) B. brevipes (Gall 51), (D) Bauhinia curvula (Gall 52), (E) B. curvula (Gall 53), (F) Bauhinia sp. (Gall 54), (G) Bauhinia sp. (Gall 55), (H) Bauhinia sp. (Gall 56), (I) Bauhinia sp. (Gall 57), (J) Bauhinia sp. (Gall 58), (K) Bauhinia sp. (Gall 59), (L) Bauhinia sp. (Gall 60), (M) Fabaceae sp. (Gall 61), (N) Fabaceae sp. (Gall 62), (0) Fabaceae sp. (Gall 63), (P) Fabaceae sp. (Gall 64), (Q) Fabaceae sp. (Gall 65), (R) Sclerolobium paniculatum (Gall 66), (S) Lamiaceae sp. (67), (T) Lamiaceae sp. (Gall 68), (U) Lauraceae sp. (Gall 69), (V) Nectandra cuspidata (Gall 70), (W) N. cuspidata (Gall 71), (X) N. cuspidata (Gall 72). 

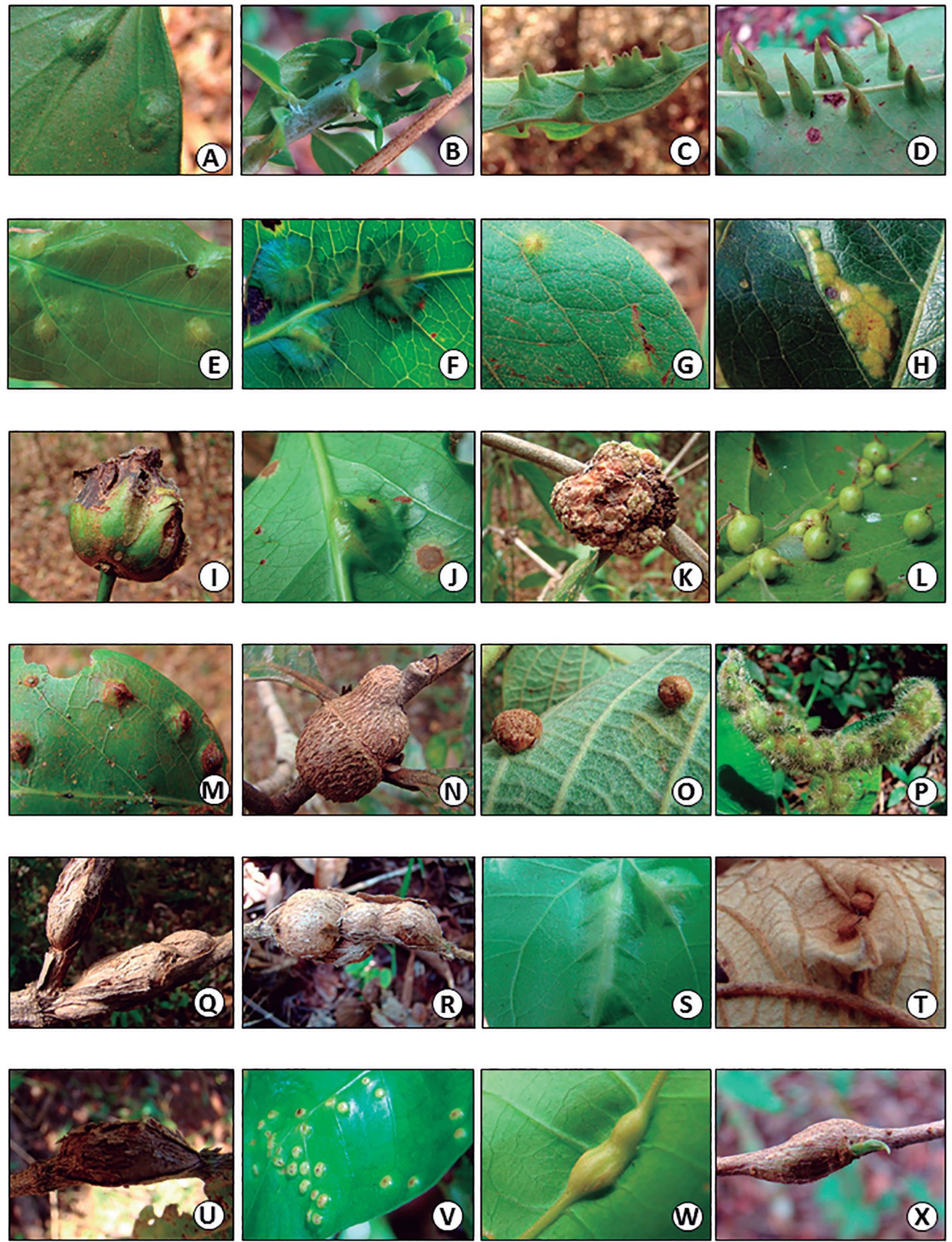

Figure 5. Insect gall morphotypes recorded in EA-CEFAEH, Hidrolândia, Goiás, Brazil. (A) Lorantaceae sp. (Gall 73), (B) Diplusodon sp. (Gall 74), (C) Byrsonima sp. (Gall 75), (D) Diplopterys pubipetala (Gall 76), (E) D. pubipetala (Gall 77), (F) D. pubipetala (Gall 78), (G) Heteropterys eglandulosa (Gall 79), (H) Heteropterys sp. (Gall 80), (I) Malpighiaceae sp. (Gall 81), (J) Malpighiaceae sp. (Gall 82), (K) Malpighiaceae sp. (Gall 83), (L) Malpighiaceae sp. (Gall 84), (M) Malpighiaceae sp. (Gall 85), (N) Malpighiaceae sp. (Gall 86), (0) Peixotoa sp. (Gall 87), (P) Melastomataceae sp. (Gall 88), (Q) Melastomataceae sp. (Gall 89), (R) Melastomataceae sp. (Gall 90), (S) Miconia sp. (Gall 91), (T) Miconia sp. (Gall 92), (U) Tibouchina sp. (Gall 93), (V) Trichilia sp. (Gall 94), (W) Trichilia sp. (Gall 95), (X) Trichilia sp. (Gall 96). 

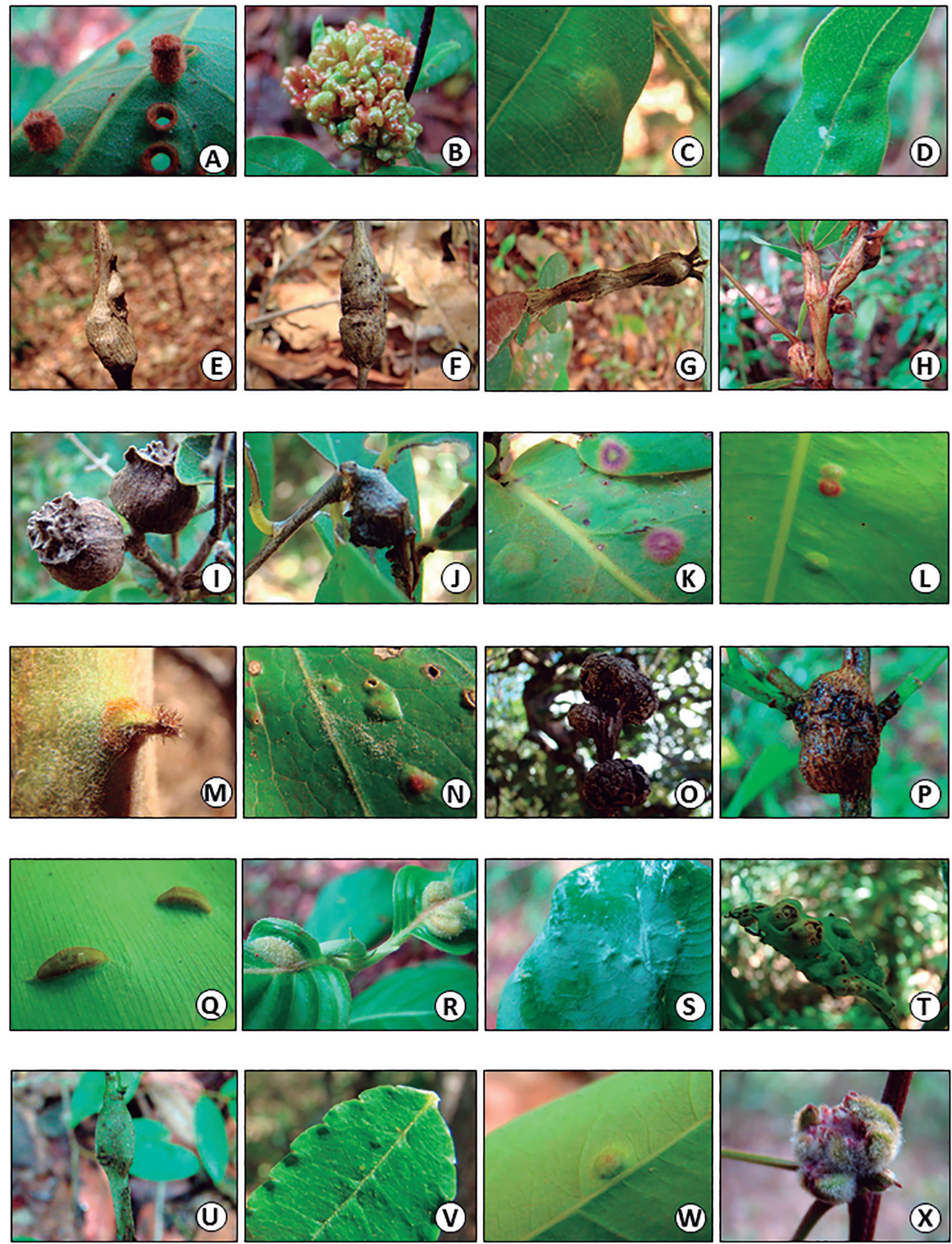

Figure 6. Insect gall morphotypes recorded in EA-CEFAEH, Hidrolândia, Goiás, Brazil. (A) Myristicaceae sp. (Gall 97), (B) Myrcia sp. (Gall 98), (C) Myrcia sp. (Gall 99), (D) Myrcia sp. (Gall 100), (E) Myrcia sp. (Gall 101), (F) Myrcia sp. (Gall 102), (G) Myrcia sp. (Gall 103), (H) Myrcia sp. (Gall 104), (I) Myrtaceae sp. (Gall 105), (J) Myrtaceae sp. (Gall 106), (K) Guapira sp. (Gall 107), (L) Ouratea hexasperma (Gall 108), (M) Roupala montana (Gall 109), (N) R. montana (Gall 110), (0) R. montana (Gall 111), (P) Rhamnidium sp. (Gall 112), (Q) Landia sp. (Gall 113), (R) Rubiaceae sp. (Gall 114), (S) Rubiaceae sp. (Gall 115), (T) Rubiaceae sp. (116), (U) Rubiaceae sp. (Gall 117), (V) Zanthoxylum sp. (Gall 118), (W) Phoradendron sp. (Gall 119), (X) Paullinia sp. (Gall 120). 

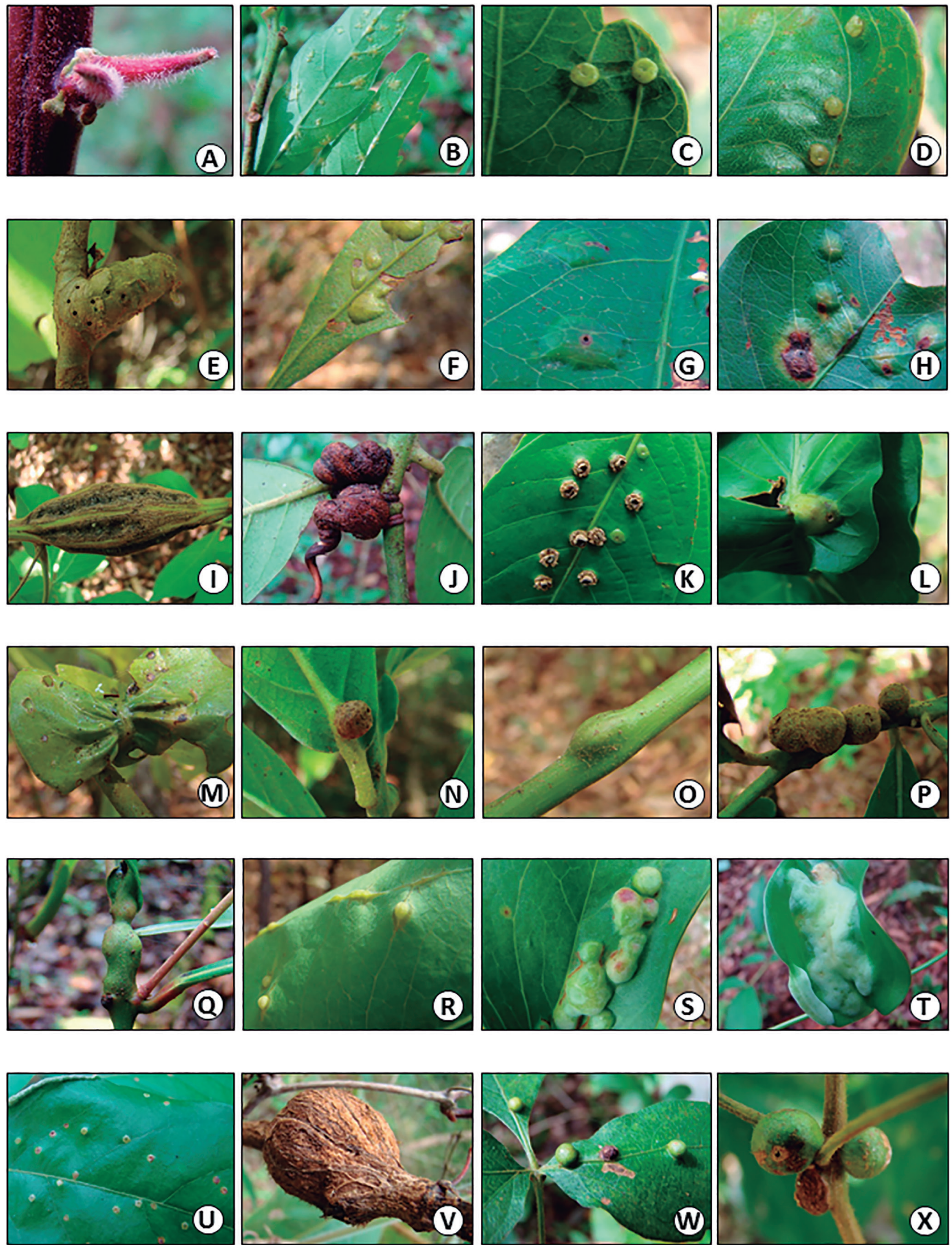

Figure 7. Insect gall morphotypes recorded in EA-CEFAEH, Hidrolândia, Goiás, Brazil. (A) Paullinia sp. (Gall 121), (B) Sapindaceae sp. (Gall 122), (C) Sapindaceae sp. (Gall 123), (D) Sapindaceae sp. (Gall 124), (E) Sapindaceae sp. (Gall 125), (F) Serjania sp. (Gall 126), (G) Serjania sp. (Gall 127), (H) Serjania sp. (Gall 128), (I) Serjania sp. (Gall 129), (J) Serjania sp. (Gall 130), (K) Siparuna guianensis (Gall 131), (L) S. guianensis (Gall 132), (M) S. guianensis (Gall 133), (N) S. guianensis (Gall 134), (0) S. guianensis (Gall 135), (P) S. guianensis (Gall 136), (Q) S. guianensis (Gall 137), (R) Smilax sp. (Gall 138), (S) Smilax sp. (Gall 139), (T) Smilax sp. (Gall 140), (U) Styrax pohlii A. DC. (Gall 141), (V) Cissus erosa (Gall 142), (W) Cissus sp. (Gall 143), (X) Vitaceae sp. (Gall 144). 

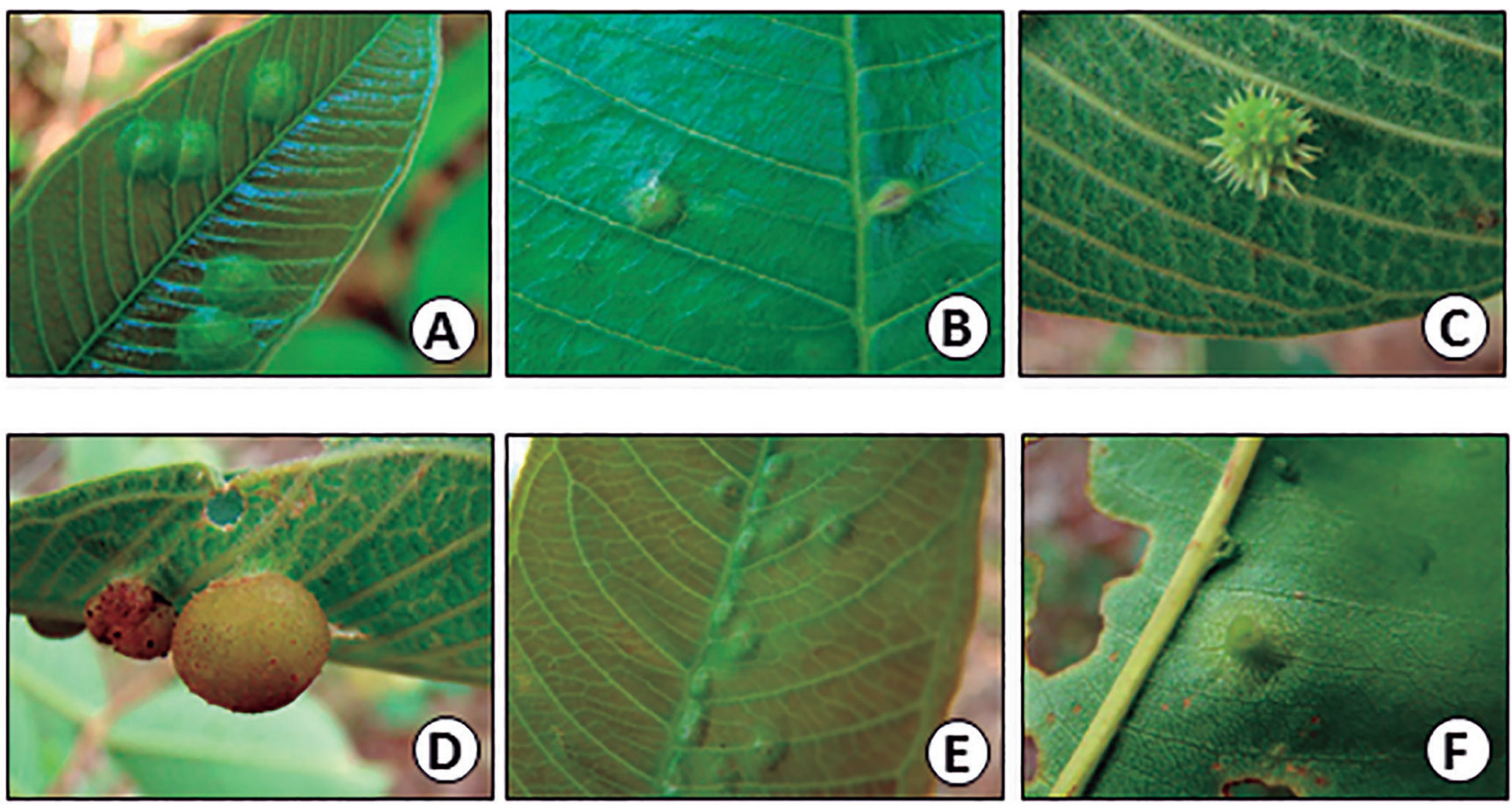

Figure 8. Insect gall morphotypes recorded in EA-CEFAEH, Hidrolândia, Goiás, Brazil. (A) Qualea dichotoma (Gall 145), (B) Qualea grandiflora (Gall 146), (C) Qualea multiflora (Gall 147), (D) Q. multiflora (Gall 148), (E) Qualea parviflora (Gall 149), (F) Q. parviflora (Gall 150).

in mesics (although it was not the objective of the work to test statistically these differences). Among the possible explanations to this pattern are that in xeric environments the plants are more nutritive to galling insects and the attack frequency of natural enemies (e.g., parasitoids) is lower as compared to mesic habitats, as predicted by hydrothermal stress hypothesis at community level (Fleck \& Fonseca, 2007; Araújo et al., 2014a).

\section{CONCLUSIONS}

This is the first systematic survey of insect galls realized in the EA-CEFAEH and region of Hidrolândia city. Although the study area is relatively anthropized and little fragments, the sampled sites presented great insect gall richness as compared to other sites cataloged to the Central region of Brazil. The EA-CEFAEH area had more insect gall morphotypes than the Parque Estadual da Serra dos Pireneus (62, Araújo et al., 2011) and Parque Nacional das Emas (97, Araújo et al., 2014b). Despite the possible effects of sampling effort differences between these studies, as previously discussed, our results show that any vegetation fragment can be a potential shelter to conserve insect galls. Finally, the observed results about host plant taxa, galling insect groups, gall morphology and occurrence between vegetation types confirm the known patterns of Brazilian Cerrado.

\section{ACKNOWLEDGEMENTS}

The authors are thankful to Julio Miguel Grandes Rios for suggestions to the manuscript; to Rodolph Delfino
Sartin for support in the project execution; to trainees of Laboratory of Entomology and Laboratory of Morphology and Plant Taxonomy (UFG) for help in the field and laboratory; to PRPPG-UFG for the grant to the first author, and the Instituto de Ciências Biológicas (UFG) for the logistical support.

\section{REFERENCES}

Araújo, W.S. 2011. Can host plant richness be used as a surrogate for galling insect diversity? Tropical Conservation Science, 4(4): 420-427.

Araújo, W.S.; Santos, B.B. \& Gomes-Klein, V.L. 2011. Insect galls from Serra dos Pireneus, G0, Brazil. Biota Neotropica, 11(2): 357-365.

Araújo, W.S.;Santos, B.B.; Guilherme, F.A.G. \& Scareli-Santos, C. 2014a. Galling insects in the Brazilian Cerrado: ecological patterns and perspectives. In: Fernandes, G.W. \& Santos, J.C. (Eds.). Neotropical Insect Galls. New York, Springer. p. 257-272.

Araújo, W.S.; Sobral, F.L. \& Maracahipes, L. 2014b. Insect galls of the Parque Nacional das Emas (Mineiros, G0, Brazil). Check List, 10(6): 1445-1451.

Carneiro, M.A.A.; Borges, R.A.X.; Araújo, A.P.A. \& Fernandes, G.W. 2009b. Insetos indutores de galhas da porção sul da Cadeia do Espinhaço, Minas Gerais, Brasil. Revista Brasileira de Entomologia, 53(4): 570-592.

Carneiro, M.A.A.; Branco, C.S.A.; Braga, C.E.D.; Almada, E.D.; Costa, M.B.M.; Maia, V.C. \& Fernandes, G.W. 2009a. Are gall midge species (Diptera, (ecidomyiidae) host-plant specialists? Revista Brasileira de Entomologia, 53(3): 365-378.

Coelho, M.S.; Fernandes, G.W.; Neves, F.S. \& Santos, J.C. 2014. Galls from Brazilian Tropical Dry Forests: Status of Knowledge and Perspectives. In: Fernandes, G.W. \& Santos, J.C. (Eds.). Neotropical Insect Galls. New York, Springer. p. 405-427.

Costa, E.C.; Carvalho-Fernandes, S.P. \& Santos-Silva, J. 2014. Galhas entomógenas associadas à Leguminosae do entorno do riacho Jatobá, Caetité, Bahia, Brasil. Revista Brasileira de Biociências, 12(2): 115-120. 
Espírito-Santo, M.M. \& Fernandes, G.W. 2007. How many species of gallinducing insects are there on Earth and where are they? Annals of Entomological Society of America, 100(2): 95-99.

Fernandes, G.W. \& Santos, J.C. 2014. Neotropical Insect Galls. New York, Springer. 550p.

Fernandes, G.W.; Tameirão-Neto, E. \& Martins, R.P. 1988. Ocorrência e caracterização de galhas entomógenas na vegetação do campus Pampulha da Universidade Federal de Minas Gerais. Revista Brasileira de Zoologia, 5(1): 11-29.

Fleck, T.\& Fonseca, C.R. 2007. Hipóteses sobre a riqueza de insetos galhadores: uma revisão considerando os níveis intra-específico, interespecífico e de comunidade. Neotropical Biology and Conservation, 2(1): 36-45.

Gagné, R. 1994. The Gall Midges of the Neotropical Region. Ithaca, Cornell University Press, 360p.

Gagné, R. 2010. Update for a catalog of the Cecidomyiidae (Diptera) of the world. Digital version 1. Washington, Entomological Society of Washington, 408p.

Gonçalves-Alvim, S.J. \& Fernandes, G.W. 2001. Biodiversity of galling insects: historical, community and habitat effects in four neotropical savannas. Biodiversity and Conservation, 10: 79-98.

Julião, G.R.;Venticinque, E.M.; Fernandes, G.W. \& Price, P.W. 2014. Unexpected High Diversity of Galling Insects in the Amazonian Upper Canopy: The Savanna Out There. PLOS ONE, 9(12): e114986.

Klink, C.A. \& Machado, R.B. 2005. Conservation of the Brazilian Cerrado. Conservation Biology, 19(3): 707-713.

Maia, V.C. \& Azevedo, M.A.P. 2009. Micro-himenópteros associados com galhas de Cecidomyiidae (Diptera) em Restingas do Estado do Rio de Janeiro (Brasil). Biota Neotropica, 9(2): 151-164.

Maia, V.C. \& Fernandes, G.W. 2004. Insect galls from Serra de São José (Tiradentes, MG, Brazil). Brazilian Journal of Biology, 6(3a): 423-445.

Maia, V.C.; Rodrigues, A.R.; Ascendino, S.H.S. \& Boggi, M. 2014. The insect gall collection of the Museu Nacional/Universidade Federal do Rio de Janeiro: biome cerrado, rupestrian fields. Brazilian Journal of Biology, 74(3): S207-S217.

Mani, MS. 1964. The Ecology of Plant Galls. The Hague, Dr. Junk. 434p.

Mendonça, R.C.; Felfili, J.M.; Walter, B.M.T.; Silva-Júnior, M.C.; Rezende, A.V.; Filgueiras, T.S.; Nogueira, P.E. \& Fagg, C.W. 2008. Flora vascular do
Bioma Cerrado: checklist com 12.356 espécies. In: Sano, S.M.; Almeida, S.P. \& Ribeiro, J.F. (Eds.). Cerrado: ecologia e flora. Brasília, EMBRAPA. p. $421-1279$.

Nogueira, R.M.; Costa, E.C.; Carvalho-Fernandes, S.P. \& Santos-Silva, J. 2016. Insect galls from Serra Geral, Caetité, BA, Brazil. Biota Neotropica, 16(1): e20150035.

Ribeiro, J.F. \& Walter, B.M.T. 2008. As principais fitofisionomias do Bioma Cerrado. In: Sano, S.M.; Almeida, S.P. \& Ribeiro, J.F. (Eds.). Cerrado: ecologia e flora. Brasília, Embrapa. p. 151-212.

Santos, B.B.; Ribeiro, B.A.; Silva, T.M. \& Araújo, W.S. 2012. Galhas de insetos em uma área de cerrado sentido restrito na região semi-urbana de Caldas Novas (Goiás, Brasil). Revista Brasileira de Biociências, 10(4): 439-445.

Santos, B.B.; Ferreira, H.D. \& Araújo, W.S. 2010. Ocorrência e caracterização de galhas em uma área de floresta estacional semidecídua em Goiânia, Goiás. Acta Botanica Brasilica, 24(1): 217-223.

Santos, J.C.; Coelho, M.S.; Souza, D.G. \& Fernandes, G.W. 2014. Galls from Brazilian Atlantic Forest: Status of Knowledge and Perspectives. In: Fernandes, G.W. \& Santos, J.C. (Eds.). Neotropical Insect Galls. New York, Springer. p. 363-376.

Shorthouse, J.D. \& Rohfritsch, 0. 1992. Biology of insect-induced galls. New York, Oxford University Press. 285p.

Silva, T.M.; Araújo, W.S. \& Santos, B.B. 2015. Ocorrência e caracterização de galhas de insetos em um fragmento de mata semicaducifólia do Campus Samambaia, Goiânia, G0, Brasil. Revista de Biologia Neotropical, 12(1): 26-38.

Southwood, T.R.E. 1960. The abundance of the Hawaiian trees and the numbers of their associated insect species. Proceedings of the Hawaiian Entomological Society, 7(2): 293-303.

Southwood, T.R.E. 1961. The number of species of insects associated with various trees. Journal of Animal Ecology, 30(1): 1-8.

Stone, G.N. \& Schönrogge, K. 2003. The adaptive significance of insect gall morphology. Trends in Ecology and Evolution, 18(10): 512-522.

Urso-Guimarães, M.V.; Scareli-Santos, C. \& Bonifácio-Silva, A.C. 2003. Occurrence and characterization of entomogen galls in plants from natural vegetation areas in Delfinópolis, MG, Brazil. Brazilian Journal of Biology, 63(4): 705-715. 